(c) 2003 American Chemical Society, Organometallics, Chan om030539g Supporting Info Page 1

Supplementary Data For:

\title{
Anionic Phosphinimine-Chelate Complexes of Rh and Ir: Steric and Electronic Influences on Oxidative Addition of $\mathrm{CH}_{2} \mathrm{Cl}_{2}$
}

Katie T. K. Chan, Liam P. Spencer, Jason D. Masuda, Jenny S. J. McCahill, Pingrong Wei, and Douglas W. Stephan* 
(C) 2003 American Chemical Society, Organometallics, Chan om030539g Supporting Info Page 2

Page 2.

Table 1. Crystal data and structure refinement for $\mathrm{Ph}_{3} \mathrm{PN}\left(2,6-\mathrm{Me}_{2} \mathrm{C}_{6} \mathrm{H}_{3}\right)$

Empirical formula

Formula weight

Temperature

Wavelength

Crystal system

Space group

Unit cell dimensions

Volume

Z

Density (calculated)

Absorption coefficient

$\mathrm{F}(000)$

Crystal size

Theta range for data collection.

Index ranges

Reflections collected

Independent reflections

Completeness to theta $=23.22^{\circ}$

Refinement method

Data / restraints / parameters

Goodness-of-fit on $\mathrm{F}^{2}$

Final $\mathrm{R}$ indices [ $\mathrm{I}>2$ sigma(I)]

$\mathrm{R}$ indices (all data)

Largest diff. peak and hole

\section{C26 H24 N P}

381.43

293(2) K

$0.71073 \AA$

monoclinic

$\mathrm{P} 2(1) / \mathrm{n}$

$a=9.626(5) \AA$

$\alpha=90^{\circ}$.

$\mathrm{b}=12.325(7) \AA$

$\beta=92.604(10)^{\circ}$.

$\mathrm{c}=17.721(9) \AA$

$\gamma=90^{\circ}$.
4

$1.206 \mathrm{Mg} / \mathrm{m}^{3}$

$0.142 \mathrm{~mm}^{-1}$

808

$0.26 \times 0.24 \times 0.20 \mathrm{~mm}^{3}$

2.01 to $23.22^{\circ}$.

$-10<=\mathrm{h}<=10,-13<=\mathrm{k}<=12,-15<=\mathrm{l}<=19$

8756

2980 [R(int) $=0.0269]$

$99.0 \%$

Full-matrix least-squares on $\mathrm{F}^{2}$

$2980 / 0 / 253$

1.023

$\mathrm{R} 1=0.0416, \mathrm{wR} 2=0.1165$

$\mathrm{R} 1=0.0552, \mathrm{wR} 2=0.1245$

0.355 and -0.301 e. $\AA^{-3}$ 
(C) 2003 American Chemical Society, Organometallics, Chan om030539g Supporting Info Page 3 Page 3

Table 2. Atomic coordinates $\left(\times 10^{4}\right)$ and equivalent isotropic displacement parameters $\left(\AA^{2} \times 10^{3}\right)$ for $\mathrm{Ph}_{3} \mathrm{PN}\left(2,6-\mathrm{Me}_{2} \mathrm{C}_{6} \mathrm{H}_{3}\right)$. U(eq) is defined as one third of the trace of the orthogonalized $\mathrm{Uii}^{\mathrm{ii}}$ tensor.

\begin{tabular}{|c|c|c|c|c|}
\hline & $\mathrm{x}$ & $y$ & $z$ & $U(e q)$ \\
\hline$P(1)$ & $1964(1)$ & $9691(1)$ & $8530(1)$ & $39(1)$ \\
\hline$N(1)$ & $1106(2)$ & $9000(1)$ & $9075(1)$ & $48(1)$ \\
\hline$C(1)$ & $2512(2)$ & $11002(2)$ & $8922(1)$ & $40(1)$ \\
\hline$C(2)$ & $3670(2)$ & $.11564(2)$ & $8672(1)$ & $48(1)$ \\
\hline$C(3)$ & $3984(3)$ & $12598(2)$ & $8937(1)$ & $55(1)$ \\
\hline$C(4)$ & $3145(3)$ & $13087(2)$ & $9449(2)$ & $57(1)$ \\
\hline$C(5)$ & $2014(3)$ & $12542(2)$ & $9707(2)$ & $60(1)$ \\
\hline$C(6)$ & $1689(3)$ & $11504(2)$ & $9448(1)$ & $51(1)$ \\
\hline$C(7)$ & $891(2)$ & $.10080(2)$ & $7699(1)$ & $41(1)$ \\
\hline$C(8)$ & $1440(3)$ & $10624(2)$ & $7086(1)$ & $51(1)$ \\
\hline$C(9)$ & $594(3)$ & $10908(2)$ & $6465(1)$ & $58(1)$ \\
\hline$C(10)$ & $-812(3)$ & $10671(2)$ & $6455(2)$ & $64(1)$ \\
\hline$C(11)$ & $-1374(3)$ & $10155(2)$ & $7060(2)$ & $63(1)$ \\
\hline$C(12)$ & $-531(2)$ & $9859(2)$ & $7680(1)$ & $50(1)$ \\
\hline$C(13)$ & $3556(2)$ & $9086(2)$ & $8201(1)$ & $41(1)$ \\
\hline$C(14)$ & $4726(2)$ & $9011(2)$ & $8696(1)$ & $52(1)$ \\
\hline$C(15)$ & $5959(3)$ & $8559(2)$ & $8467(2)$ & $61(1)$ \\
\hline$C(16)$ & $6047(3)$ & $8174(2)$ & $7742(2)$ & $59(1)$ \\
\hline$C(17)$ & $4895(3)$ & $8216(2)$ & $7252(2)$ & $59(1)$ \\
\hline$\dot{C}(18)$ & $3658(3)$ & $8666(2)$ & $7477(1)$ & $51(1)$ \\
\hline$C(19)$ & $1352(2)$ & $7988(2)$ & $9417(1)$ & $41(1)$ \\
\hline$C(20)$ & $877(2)$ & $7025(2)$ & $9057(1)$ & $47(1)$ \\
\hline$C(21)$ & $1060(3)$ & $6030(2)$ & $9421(2)$ & $61(1)$ \\
\hline$C(22)$ & $1683(3)$ & $5963(2)$ & $10135(2)$ & $70(1)$ \\
\hline$C(23)$ & $2125(3)$ & $6899(2)$ & $10492(2)$ & $65(1)$ \\
\hline$C(24)$ & $1981(2)$ & $7917(2)$ & $10151(1)$ & $51(1)$ \\
\hline$C(25)$ & $130(3)$ & $7075(2)$ & $8291(2)$ & $66(1)$ \\
\hline$C(26)$ & $2449(3)$ & $8924(2)$ & $10564(2)$ & $71(1)$ \\
\hline
\end{tabular}


(C) 2003 American Chemical Society, Organometallics, Chan om030539g Supporting Info Page 4 Page 4

Table 3. Bond lengths $[\AA]$ and angles $\left[^{\circ}\right]$ for $\mathrm{Ph}_{3} \mathrm{PN}\left(2,6-\mathrm{Me}_{2} \mathrm{C}_{6} \mathrm{H}_{3}\right)$.

\begin{tabular}{|c|c|c|}
\hline$P(1)-N(1)$ & $1.553(2)$ & \\
\hline$P(1)-C(13)$ & $1.824(2)$ & \\
\hline$P(1)-C(7)$ & $1.824(2)$ & \\
\hline$P(1)-C(1)$ & $1.826(2)$ & \\
\hline$N(1)-C(19)$ & $1.403(3)$ & \\
\hline$C(1)-C(6)$ & $1.395(3)$ & \\
\hline$C(1)-C(2)$ & $1.402(3)$ & \\
\hline $\mathrm{C}(2)-\mathrm{C}(3)$ & $1.387(3)$ & \\
\hline$C(3)-C(4)$ & $1.380(4)$ & \\
\hline$C(4)-C(5)$ & $1.374(4)$ & \\
\hline$C(5)-C(6)$ & $1.390(3)$ & \\
\hline$C(7)-C(12)$ & $1.394(3)$ & \\
\hline$C(7)-C(8)$ & $1.402(3)$ & \\
\hline$C(8)-C(9)$ & $1.383(4)$ & \\
\hline$C(9)-C(10)$ & $1.384(4)$ & \\
\hline$C(10)-C(11)$ & $1.377(4)$ & \\
\hline$C(11)-C(12)$ & $1.386(3)$ & \\
\hline$C(13)-C(18)$ & $1.391(3)$ & \\
\hline$C(13)-C(14)$ & $1.399(3)$ & \\
\hline$C(14)-C(15)$ & $1.389(3)$ & \\
\hline$C(15)-C(16)$ & $1.376(4)$ & \\
\hline$C(16)-C(17)$ & $1.379(4)$ & \\
\hline$C(17)-C(18)$ & $1.389(4)$ & \\
\hline$C(19)-C(20)$ & $1.413(3)$ & . \\
\hline$C(19)-C(24)$ & $1.413(3)$ & \\
\hline$C(20)-C(21)$ & $1.393(3)$ & \\
\hline$C(20)-C(25)$ & $1.508(4)$ & \\
\hline$C(21)-C(22)$ & $1.378(4)$ & \\
\hline$C(22)-C(23)$ & $1.373(4)$ & \\
\hline$C(23)-C(24)$ & $1.396(3)$ & \\
\hline$C(24)-C(26)$ & $1.500(4)$ & \\
\hline$N(1)-P(1)-C(13)$ & $116.89(10)$ & \\
\hline$N(1)-P(1)-C(7)$ & $110.35(11)$ & \\
\hline$C(13)-P(1)-C(7)$ & $107.57(11)$ & \\
\hline
\end{tabular}


(C) 2003 American Chemical Society, Organometallics, Chan om030539g Supporting Info Page 5

\begin{tabular}{|c|c|}
\hline$N(1)-P(1)-C(1)$ & $113.68(10)$ \\
\hline$C(13)-P(1)-C(1)$ & $104.59(10)$ \\
\hline$C(7)-P(1)-C(1)$ & $102.60(10)$ \\
\hline$C(19)-N(1)-P(1)$ & $132.31(16)$ \\
\hline$C(6)-C(1)-C(2)$ & $118.4(2)$ \\
\hline$C(6)-C(1)-P(1)$ & $118.94(17)$ \\
\hline$C(2)-C(1)-P(1)$ & $122.44(16)$ \\
\hline$C(3)-C(2)-C(1)$ & $120.7(2)$ \\
\hline$C(4)-C(3)-C(2)$ & $120.0(2)$ \\
\hline$C(5)-C(4)-C(3)$ & $120.1(2)$ \\
\hline$C(4)-C(5)-C(6)$ & $120.7(2)$ \\
\hline$C(5)-C(6)-C(1)$ & $120.2(2)$ \\
\hline$C(12)-C(7)-C(8)$ & $118.6(2)$ \\
\hline$C(12)-C(7)-P(1)$ & $119.20(17)$ \\
\hline$C(8)-C(7)-P(1)$ & $122.15(17)$ \\
\hline$C(9)-C(8)-C(7)$ & $120.6(2)$ \\
\hline$C(8)-C(9)-C(10)$ & $119.8(2)$ \\
\hline$C(11)-C(10)-C(9)$ & $120.4(2)$ \\
\hline$C(10)-C(11)-C(12)$ & $120.1(3)$ \\
\hline$C(11)-C(12)-C(7)$ & $120.5(2)$ \\
\hline$C(18)-C(13)-C(14)$ & $117.7(2)$ \\
\hline$C(18)-C(13)-P(1)$ & $122.90(17)$ \\
\hline$C(14)-C(13)-P(1)$ & $119.34(18)$ \\
\hline$C(15)-C(14)-C(13)$ & $121.2(2)$ \\
\hline$C(16)-C(15)-C(14)$ & $120.0(2)$ \\
\hline$C(15)-C(16)-C(17)$ & $119.6(2)$ \\
\hline$C(16)-C(17)-C(18)$ & $120.6(2)$ \\
\hline$C(17)-C(18)-C(13)$ & $120.7(2)$ \\
\hline$N(1)-C(19)-C(20)$ & $120.4(2)$ \\
\hline$N(1)-C(19)-C(24)$ & $120.7(2)$ \\
\hline$C(20)-C(19)-C(24)$ & $118.7(2)$ \\
\hline$C(21)-C(20)-C(19)$ & $119.9(2)$ \\
\hline$C(21)-C(20)-C(25)$ & $119.8(2)$ \\
\hline$C(19)-C(20)-C(25)$ & $120.3(2)$ \\
\hline$C(22)-C(21)-C(20)$ & $121.2(3)$ \\
\hline$C(23)-C(22)-C(21)$ & $119.0(2)$ \\
\hline
\end{tabular}


(C) 2003 American Chemical Society, Organometallics, Chan om030539g Supporting Info Page 6

$\begin{array}{ll}\mathrm{C}(22)-\mathrm{C}(23)-\mathrm{C}(24) & 122.2(3) \\ \mathrm{C}(23)-\mathrm{C}(24)-\mathrm{C}(19) & 118.9(2) \\ \mathrm{C}(23)-\mathrm{C}(24)-\mathrm{C}(26) & 120.8(2) \\ \mathrm{C}(19)-\mathrm{C}(24)-\mathrm{C}(26) & 120.3(2)\end{array}$

Symmetry transformations used to generate equivalent atoms:

Table 4. Anisotropic displacement parameters $\left(\AA^{2} \times 10^{3}\right)$ for $\mathrm{Ph}_{3} \mathrm{PN}\left(2,6-\mathrm{Me}_{2} \mathrm{C}_{6} \mathrm{H}_{3}\right)$. The anisotropic displacement factor exponent takes the form: $-2 \pi^{2}\left[h^{2} a^{* 2} U^{11}+\ldots+2 h k a^{*} b^{*} U^{12}\right]$

\begin{tabular}{|c|c|c|c|c|c|c|}
\hline & $\mathrm{U}^{11}$ & $\mathrm{U}^{22}$ & $\mathrm{U}^{33}$ & $\mathrm{U}^{23}$ & $U^{13}$ & $\mathrm{U}^{12}$ \\
\hline$P(1)$ & $36(1)$ & $4 \cdot 1(1)$ & $40(1)$ & $-1(1)$ & $1(1)$ & $-2(1)$ \\
\hline $\mathrm{N}(1)$ & $45(1)$ & $48(1)$ & $52(1)$ & $7(1)$ & $8(1)$ & $0(1)$ \\
\hline$C(1)$ & $37(1)$ & $43(1)$ & $38(1)$ & $2(1)$ & $-2(1)$ & $-1(1)$ \\
\hline$C(2)$ & $46(1)$ & $47(1)$ & $52(2)$ & $-5(1)$ & $8(1)$ & $-2(1)$ \\
\hline$C(3)$ & $52(2)$ & $49(1)$ & $65(2)$ & $-2(1)$ & $4(1)$ & $-10(1)$ \\
\hline$C(4)$ & $70(2)$ & $44(1)$ & $57(2)$ & $-10(1)$ & $-3(1)$ & $-5(1)$ \\
\hline$C(5)$ & $67(2)$ & $57(2)$ & $58(2)$ & $-15(1)$ & $13(1)$ & $4(1)$ \\
\hline$C(6)$ & $46(1)$ & $55(1)$ & $52(2)$ & $-5(1)$ & $8(1)$ & $0(1)$ \\
\hline$C(7)$ & $41(1)$ & $40(1)$ & $42(1)$ & $-3(1)$ & $-1(1)$ & $-2(1)$ \\
\hline$C(8)$ & $46(1)$ & $53(1)$ & $52(2)$ & $5(1)$ & $2(1)$ & $-4(1)$ \\
\hline$C(9)$ & $68(2)$ & $58(2)$ & $47(2)$ & $9(1)$ & $O(1)$ & $-1(1)$ \\
\hline$C(10)$ & $70(2)$ & $61(2)$ & $58(2)$ & $8(1)$ & $-20(1)$ & $-1(1)$ \\
\hline$C(11)$ & $47(2)$ & $63(2)$ & $78(2)$ & $8(1)$ & $-16(1)$ & $-7(1)$ \\
\hline$C(12)$ & $45(1)$ & $50(1)$ & $55(2)$ & $7(1)$ & $-2(1)$ & $-5(1)$ \\
\hline$C(13)$ & $40(1)$ & $36(1)$ & $46(1)$ & $-2(1)$ & $1(1)$ & $-3(1)$ \\
\hline$C(14)$ & $48(2)$ & $55(1)$ & $52(2)$ & $-6(1)$ & $-2(1)$ & $5(1)$ \\
\hline$C(15)$ & $46(2)$ & $60(2)$ & $75(2)$ & $-4(1)$ & $-8(1)$ & $6(1)$ \\
\hline$C(16)$ & $47(2)$ & $56(1)$ & $75(2)$ & $-5(1)$ & $14(1)$ & $4(1)$ \\
\hline$C(17)$ & $59(2)$ & $62(2)$ & $58(2)$ & $-10(1)$ & $14(1)$ & $2(1)$ \\
\hline$C(18)$ & $46(2)$ & $56(1)$ & $49(2)$ & $-8(1)$ & $0(1)$ & $0(1)$ \\
\hline$C(19)$ & $33(1)$ & $47(1)$ & $45(1)$ & $3(1)$ & $10(1)$ & $1(1)$ \\
\hline $\mathrm{C}(20)$ & $41(1)$ & $48(1)$ & $53(2)$ & $-3(1)$ & $13(1)$ & $-1(1)$ \\
\hline$C(21)$ & $54(2)$ & $46(1)$ & $83(2)$ & $0(1)$ & $17(2)$ & $1(1)$ \\
\hline$C(22)$ & $63(2)$ & $58(2)$ & $90(2)$ & $25(2)$ & $13(2)$ & $7(1)$ \\
\hline
\end{tabular}


(C) 2003 American Chemical Society, Organometallics, Chan om030539g Supporting Info Page 7

\begin{tabular}{lllllll}
$\mathrm{C}(23)$ & $55(2)$ & $82(2)$ & $57(2)$ & $24(2)$ & $4(1)$ & $6(1)$ \\
$\mathrm{C}(24)$ & $42(1)$ & $63(2)$ & $47(2)$ & $5(1)$ & $5(1)$ & $-2(1)$ \\
$\mathrm{C}(25)$ & $65(2)$ & $66(2)$ & $68(2)$ & $-16(1)$ & $-4(1)$ & $-4(1)$ \\
$\mathrm{C}(26)$ & $69(2)$ & $87(2)$ & $56(2)$ & $-3(2)$ & $-10(1)$ & $-9(2)$ \\
\hline
\end{tabular}

Table 5. Hydrogen coordinates ( $\left.\times 10^{4}\right)$ and isotropic displacement parameters $\left(\AA^{2} \times 10^{3}\right)$ for $\mathrm{Ph}_{3} \mathrm{PN}\left(2,6-\mathrm{Me}_{2} \mathrm{C}_{6} \mathrm{H}_{3}\right)$.

\begin{tabular}{|c|c|c|c|c|}
\hline & $\mathrm{x}$ & $\mathrm{y}$ & $\mathrm{z}$ & $\mathrm{U}(\mathrm{eq})$ \\
\hline $\mathrm{H}(2 \mathrm{~A})$ & 4234 & 11241 & 8323 & 58 \\
\hline$H(3 A)$ & 4759 & 12961 & 8771 & 66 \\
\hline $\mathrm{H}(4 \mathrm{~A})$ & 3345 & 13785 & 9620 & 69 \\
\hline $\mathrm{H}(5 \mathrm{~A})$ & 1461 & 12872 & 10058 & 72 \\
\hline$H(6 A)$ & 921 & 11144 & 9626 & 61 \\
\hline $\mathrm{H}(8 \mathrm{~A})$ & 2382 & 10794 & 7095 & 61 \\
\hline $\mathrm{H}(9 \mathrm{~A})$ & 969 & 11258 & 6056 & 69 \\
\hline $\mathrm{H}(10 \mathrm{~A})$ & -1381 & 10860 & 6038 & 77 \\
\hline $\mathrm{H}(11 \mathrm{~A})$ & -2321 & 10005 & 7052 & 76 \\
\hline $\mathrm{H}(12 \mathrm{~A})$ & -915 & 9510 & 8087 & 60 \\
\hline $\mathrm{H}(14 \mathrm{~A})$ & 4677 & 9269 & 9187 & 62 \\
\hline$H(15 A)$ & 6726 & 8516 & 8804 & 73 \\
\hline$H(16 A)$ & 6879 & 7888 & 7583 & 71 \\
\hline $\mathrm{H}(17 \mathrm{~A})$ & 4947 & 7940 & 6765 & 71 \\
\hline $\mathrm{H}(18 \mathrm{~A})$ & 2889 & 8686 & 7140 & 61 \\
\hline $\mathrm{H}(21 \mathrm{~A})$ & 756 & 5399 & 9178 & 73 \\
\hline $\mathrm{H}(22 \mathrm{~A})$ & 1802 & 5295 & 10372 & 84 \\
\hline $\mathrm{H}(23 \mathrm{~A})$ & 2533 & 6853 & 10977 & 78 \\
\hline $\mathrm{H}(25 \mathrm{~A})$ & 92 & 7814 & 8119 & 99 \\
\hline $\mathrm{H}(25 \mathrm{~B})$ & -797 & 6800 & 8326 & 99 \\
\hline $\mathrm{H}(25 \mathrm{C})$ & 621 & 6643 & 7939 & 99 \\
\hline $\mathrm{H}(26 \mathrm{~A})$ & 2844 & 8732 & 11053 & 107 \\
\hline$H(26 B)$ & 1666 & 9395 & 10622 & 107 \\
\hline$H(26 C)$ & 3135 & 9291 & 10281 & 107 \\
\hline
\end{tabular}


Table 1. Crystal data and structure refinement for $\mathrm{Rh}(\mathrm{COD})\left(\mathrm{O}-\mathrm{C}_{6} \mathrm{H}_{4} \mathrm{PPh}_{2} \mathrm{NPh}\right)$.

Empirical formula

Formula weight

Temperature

Wavelength

Crystal system

Space group

Unit cell dimensions

Volume

Z

Density (calculated)

Absorption coefficient

$\mathrm{F}(000)$

Crystal size

Theta range for data collection

Index ranges

Reflections collected

Independent reflections

Completeness to theta $=23.24^{\circ}$

Refinement method

Data / restraints / parameters

Goodness-of-fit on $\mathrm{F}^{2}$

Final $R$ indices [ $\mathrm{I}>2 \operatorname{sigma}(\mathrm{I})]$

$R$ indices (all data)

Largest diff. peak and hole
C32 H31 N P Rh

563.46

293(2) K

$0.71073 \AA$

trinclinic

P-1

$\mathrm{a}=9.835(5) \AA$

$\alpha=79.137(10)^{\circ}$.

$b=11.331(6) \AA$

$\beta=88.477(10)^{\circ}$.

$\mathrm{c}=12.325(7) \AA$

$\gamma=77.591(10)^{\circ}$.
2

$1.421 \mathrm{Mg} / \mathrm{m}^{3}$

$0.730 \mathrm{~mm}^{-1}$

580 .

$0.32 \times 0.23 \times 0.21 \mathrm{~mm}^{3}$

1.87 to $23.24^{\circ}$.

$-10<=\mathrm{h}<=8,-12<=\mathrm{k}<=11,-13<=\mathrm{k}<=13$

5636

$3736[\mathrm{R}(\mathrm{int})=0.0149]$

$98.5 \%$

Full-matrix least-squares on $\mathrm{F}^{2}$

$3736 / 0 / 316$

0.982

$\mathrm{R} 1=0.0251, \mathrm{wR} 2=0.0683$

$\mathrm{R} I=0.0283, \mathrm{wR} 2=0.0693$

0.425 and -0.444 e. $\AA^{-3}$ 
(C) 2003 American Chemical Society, Organometallics, Chan om030539g Supporting Info Page 9

Table 2. Atomic coordinates $\left(\times 10^{4}\right)$ and equivalent isotropic displacement parameters $\left(\AA^{2} \times 10^{3}\right)$ for $\mathrm{Rh}(\mathrm{COD})\left(\mathrm{o}-\mathrm{C}_{6} \mathrm{H}_{4} \mathrm{PPh}_{2} \mathrm{NPh}\right)$. U(eq) is defined as one third of the trace of the orthogonalized $\mathrm{U}^{\mathrm{ij}}$ tensor.

\begin{tabular}{|c|c|c|c|c|}
\hline & $\mathrm{x}$ & $\mathrm{y}$ & $z$ & $\mathrm{U}(\mathrm{eq})$ \\
\hline $\operatorname{Rh}(1)$ & $7080(1)$ & $5778(1)$ & $7367(1)$ & $35(1)$ \\
\hline$N(1)$ & $7649(2)$ & $7248(2)$ & $7946(2)$ & $41(1)$ \\
\hline$P(1)$ & $8206(1)$ & $8227(1)$ & $7016(1)$ & $35(1)$ \\
\hline$C(1)$ & $8083(3)$ & $6377(2)$ & $5936(2)$ & $37(1)$ \\
\hline$C(2)$ & $8321(3)$ & $5843(3)$ & $4986(2)$ & $44(1)$ \\
\hline$C(3)$ & $8938(3)$ & $6376(3)$ & $4046(2)$ & $50(1)$ \\
\hline$C(4)$ & $9340(3)$ & $7481(3)$ & $3993(2)$ & $52(1)$ \\
\hline$C(5)$ & $9139(3)$ & $8050(3)$ & $4889(2)$ & $47(1)$ \\
\hline $\mathrm{C}(6)$ & $8541(3)$ & $7496(2)$ & $5843(2)$ & $37(1)$ \\
\hline$C(7)$ & $6952(3)$ & $9669(2)$ & $6568(2)$ & $41(1)$ \\
\hline$C(8)$ & $.7369(3)$ & $10741(3)$ & $6064(2)$ & $55(1)$ \\
\hline $\mathrm{C}(9)$ & $6374(5)$ & $11780(3)$ & $5629(3)$ & $74(1)$ \\
\hline$C(10)$ & $4981(5)$ & $11766(4)$ & $5700(3)$ & $83(1)$ \\
\hline$C(11)$ & $4569(4)$ & $10708(4)$ & $6184(3)$ & $78(1)$ \\
\hline$C(12)$ & $5547(3)$ & $9651(3)$ & $6617(2)$ & $55(1)$ \\
\hline$C(13)$ & $9750(3)$ & $8658(2)$ & $7450(2)$ & $40(1)$ \\
\hline$C(14)$ & $9627(3)$ & $9509(3)$ & $8151(2)$ & $49(1)$ \\
\hline$C(15)$ & $10816(4)$ & $9771(3)$ & $8555(3)$ & $62(1)$ \\
\hline$C(16)$ & $12116(4)$ & $9184(3)$ & $8255(3)$ & $71(1)$ \\
\hline$C(17)$ & $12244(3)$ & $8346(4)$ & $7584(3)$ & $74(1)$ \\
\hline$C(18)$ & $11074(3)$ & $8073(3)$ & $7172(3)$ & $59(1)$ \\
\hline$C(19)$ & $7557(3)$ & $7455(2)$ & $9070(2)$ & $41(1)$ \\
\hline$C(20)$ & $8632(3)$ & $6865(3)$ & $9809(2)$ & $55(1)$ \\
\hline$C(21)$ & $8543(4)$ & $7002(4)$ & $10911(2)$ & $67(1)$ \\
\hline$C(22)$ & $7385(4)$ & $7730(3)$ & $11272(2)$ & $66(1)$ \\
\hline$C(23)$ & $6321(4)$ & $8328(3)$ & $10554(3)$ & $61(1)$ \\
\hline$C(24)$ & $6396(3)$ & $8191(3)$ & $9440(2)$ & $52(1)$ \\
\hline$C(25)$ & $5894(3)$ & $4894(3)$ & $6525(2)$ & $47(1)$ \\
\hline$C(26)$ & $4423(3)$ & $4988(4)$ & $6974(3)$ & $64(1)$ \\
\hline$C(27)$ & $4317(3)$ & $5019(3)$ & $8186(3)$ & $57(1)$ \\
\hline
\end{tabular}


(C) 2003 American Chemical Society, Organometallics, Chan om030539g Supporting Info Page 10 Page 10

$\begin{array}{lllll}\mathrm{C}(28) & 5452(3) & 5523(3) & 8625(2) & 47(1) \\ \mathrm{C}(29) & 6708(3) & 4804(3) & 9038(2) & 51(1) \\ \mathrm{C}(30) & 7153(4) & 3431(3) & 9089(3) & 65(1) \\ \mathrm{C}(31) & 7027(5) & 3032(3) & 7999(3) & 72(1) \\ \mathrm{C}(32) & 7089(3) & 4038(3) & 6991(2) & 50(1)\end{array}$

Table 3. Bond lengths $[\AA]$ and angles $\left[^{\circ}\right]$ for $R h(C O D)\left(O-C_{6} H_{4} P P h_{2} N P h\right)$.

\begin{tabular}{ll}
\hline $\mathrm{Rh}(1)-\mathrm{C}(1)$ & $2.071(3)$ \\
$\mathrm{Rh}(1)-\mathrm{C}(25)$ & $2.097(3)$ \\
$\mathrm{Rh}(1)-\mathrm{C}(32)$ & $2.106(3)$ \\
$\mathrm{Rh}(1)-\mathrm{N}(1)$ & $2.114(2)$ \\
$\mathrm{Rh}(1)-\mathrm{C}(29)$ & $2.207(3)$ \\
$\mathrm{Rh}(1)-\mathrm{C}(28)$ & $2.225(3)$ \\
$\mathrm{N}(1)-\mathrm{C}(19)$ & $1.445(3)$ \\
$\mathrm{N}(1)-\mathrm{P}(1)$ & $1.610(2)$ \\
$\mathrm{P}(1)-\mathrm{C}(6)$ & $1.786(3)$ \\
$\mathrm{P}(1)-\mathrm{C}(13)$ & $1.815(3)$ \\
$\mathrm{P}(1)-\mathrm{C}(7)$ & $1.823(3)$ \\
$\mathrm{C}(1)-\mathrm{C}(2)$ & $1.409(4)$ \\
$\mathrm{C}(1)-\mathrm{C}(6)$ & $1.419(4)$ \\
$\mathrm{C}(2)-\mathrm{C}(3)$ & $1.389(4)$ \\
$\mathrm{C}(3)-\mathrm{C}(4)$ & $1.383(4)$ \\
$\mathrm{C}(4)-\mathrm{C}(5)$ & $1.370(4)$ \\
$\mathrm{C}(5)-\mathrm{C}(6)$ & $1.402(4)$ \\
$\mathrm{C}(7)-\mathrm{C}(12)$ & $1.385(4)$ \\
$\mathrm{C}(7)-\mathrm{C}(8)$ & $1.395(4)$ \\
$\mathrm{C}(8)-\mathrm{C}(9)$ & $1.388(5)$ \\
$\mathrm{C}(9)-\mathrm{C}(10)$ & $1.374(6)$ \\
$\mathrm{C}(10)-\mathrm{C}(11)$ & $1.372(6)$ \\
$\mathrm{C}(11)-\mathrm{C}(12)$ & $1.392(5)$ \\
$\mathrm{C}(13)-\mathrm{C}(18)$ & $1.393(4)$ \\
$\mathrm{C}(13)-\mathrm{C}(14)$ & $1.396(4)$ \\
$\mathrm{C}(14)-\mathrm{C}(15)$ & $1.393(4)$ \\
$\mathrm{C}(15)-\mathrm{C}(16)$ & \\
$\mathrm{C}(16)-\mathrm{C}(17)$ & \\
& $1.385(5)$ \\
&
\end{tabular}


(C) 2003 American Chemical Society, Organometallics, Chan om030539g Supporting Info Page 11

\begin{tabular}{|c|c|}
\hline$C(17)-C(18)$ & $1.386(5)$ \\
\hline$C(19)-C(24)$ & $1.384(4)$ \\
\hline$C(19)-C(20)$ & $1.382(4)$ \\
\hline$C(20)-C(21)$ & $1.395(4)$ \\
\hline$C(21)-C(22)$ & $1.372(5)$ \\
\hline$C(22)-C(23)$ & $1.362(5)$ \\
\hline$C(23)-C(24)$ & $1.408(4)$ \\
\hline$C(25)-C(32)$ & $1.410(4)$ \\
\hline$C(25)-C(26)$ & $1.525(4)$ \\
\hline$C(26)-C(27)$ & $1.501(4)$ \\
\hline$C(27)-C(28)$ & $1.513(4)$ \\
\hline$C(28)-C(29)$ & $1.376(4)$ \\
\hline$C(29)-C(30)$ & $1.512(4)$ \\
\hline$C(30)-C(31)$ & $1.512(5)$ \\
\hline$C(31)-C(32)$ & $1.530(4)$ \\
\hline$C(1)-\operatorname{Rh}(1)-C(25)$ & $92.05(11)$ \\
\hline$C(1)-\operatorname{Rh}(1)-C(32)$ & $94.55(10)$ \\
\hline$C(25)-R h(1)-C(32)$ & $39.21(12)$ \\
\hline$C(1)-R h(1)-N(1)$ & $84.90(9)$ \\
\hline$C(25)-R h(1)-N(1)$ & $157.55(11)$ \\
\hline$C(32)-\operatorname{Rh}(1)-\mathrm{N}(1)$ & $163.15(11)$ \\
\hline$C(1)-\operatorname{Rh}(1)-C(29)$ & $161.29(11)$ \\
\hline$C(25)-\operatorname{Rh}(1)-C(29)$ & $95.92(11)$ \\
\hline$C(32)-R h(1)-C(29)$ & $81.26(11)$ \\
\hline$N(1)-\operatorname{Rh}(1)-C(29)$ & $93.86(10)$ \\
\hline$C(1)-\operatorname{Rh}(1)-C(28)$ & $162.52(10)$ \\
\hline$C(25)-\operatorname{Rh}(1)-C(28)$ & $81.07(11)$ \\
\hline $\mathrm{C}(32)-\mathrm{Rh}(1)-\mathrm{C}(28)$ & $90.22(11)$ \\
\hline $\mathrm{N}(1)-\mathrm{Rh}(1)-\mathrm{C}(28)$ & $95.31(10)$ \\
\hline$C(29)-R h(1)-C(28)$ & $36.18(11)$ \\
\hline$C(19)-N(1)-P(1)$ & $118.50(17)$ \\
\hline$C(19)-N(1)-R h(1)$ & $126.32(16)$ \\
\hline $\mathrm{P}(1)-\mathrm{N}(1)-\mathrm{Rh}(1)$ & $115.17(12)$ \\
\hline$N(1)-P(1)-C(6)$ & $105.03(12)$ \\
\hline$N(1)-P(1)-C(13)$ & $113.13(12)$ \\
\hline$C(6)-P(1)-C(13)$ & $111.39(12)$ \\
\hline
\end{tabular}


(C) 2003 American Chemical Society, Organometallics, Chan om030539g Supporting Info Page 12

\begin{tabular}{|c|c|}
\hline$N(1)-P(1)-C(7)$ & $114.72(13)$ \\
\hline$C(6)-P(1)-C(7)$ & $106.83(12)$ \\
\hline$C(13)-P(1)-C(7)$ & $105.67(13)$ \\
\hline$C(2)-C(1)-C(6)$ & $113.8(2)$ \\
\hline$C(2)-C(1)-R h(1)$ & $128.1(2)$ \\
\hline$C(6)-C(1)-R h(1)$ & $118.05(18)$ \\
\hline$C(3)-C(2)-C(1)$ & $122.7(3)$ \\
\hline$C(4)-C(3)-C(2)$ & $121.1(3)$ \\
\hline$C(5)-C(4)-C(3)$ & $119.1(2)$ \\
\hline$C(4)-C(5)-C(6)$ & $119.5(3)$ \\
\hline$C(5)-C(6)-C(1)$ & $123.7(2)$ \\
\hline$C(5)-C(6)-P(1)$ & $121.1(2)$ \\
\hline$C(1)-C(6)-P(1)$ & $115.05(18)$ \\
\hline$C(12)-C(7)-C(8)$ & $119.5(3)$ \\
\hline$C(12)-C(7)-P(1)$ & $118.3(2)$ \\
\hline$C(8)-C(7)-P(1)$ & $121.8(2)$ \\
\hline$C(9)-C(8)-C(7)$ & $119.8(3)$ \\
\hline$C(10)-C(9)-C(8)$ & $120.5(4)$ \\
\hline$C(11)-C(10)-C(9)$ & $119.8(3)$ \\
\hline$C(10)-C(11)-C(12)$ & $120.7(4)$ \\
\hline$C(7)-C(12)-C(11)$ & $119.7(3)$ \\
\hline$C(18)-C(13)-C(14)$ & $119.1(3)$ \\
\hline$C(18)-C(13)-P(1)$ & $120.8(2)$ \\
\hline$C(14)-C(13)-P(1)$ & $119.8(2)$ \\
\hline$C(15)-C(14)-C(13)$ & $120.0(3)$ \\
\hline$C(16)-C(15)-C(14)$ & $119.5(3)$ \\
\hline$C(17)-C(16)-C(15)$ & $120.7(3)$ \\
\hline$C(16)-C(17)-C(18)$ & $120.6(3)$ \\
\hline$C(17)-C(18)-C(13)$ & $120.0(3)$ \\
\hline$C(24)-C(19)-C(20)$ & $118.9(3)$ \\
\hline$C(24)-C(19)-N(1)$ & $121.7(2)$ \\
\hline$C(20)-C(19)-N(1)$ & $119.3(3)$ \\
\hline$C(19)-C(20)-C(21)$ & $120.6(3)$ \\
\hline$C(22)-C(21)-C(20)$ & $120.1(3)$ \\
\hline$C(23)-C(22)-C(21)$ & $120.2(3)$ \\
\hline$C(22)-C(23)-C(24)$ & $120.2(3)$ \\
\hline
\end{tabular}


(C) 2003 American Chemical Society, Organometallics, Chan om030539g Supporting Info Page 13

$\begin{array}{lc}\mathrm{C}(19)-\mathrm{C}(24)-\mathrm{C}(23) & 120.0(3) \\ \mathrm{C}(32)-\mathrm{C}(25)-\mathrm{C}(26) & 125.6(3) \\ \mathrm{C}(32)-\mathrm{C}(25)-\mathrm{Rh}(1) & 70.75(16) \\ \mathrm{C}(26)-\mathrm{C}(25)-\mathrm{Rh}(1) & 111.84(19) \\ \mathrm{C}(27)-\mathrm{C}(26)-\mathrm{C}(25) & 114.3(3) \\ \mathrm{C}(26)-\mathrm{C}(27)-\mathrm{C}(28) & 113.8(2) \\ \mathrm{C}(29)-\mathrm{C}(28)-\mathrm{C}(27) & 123.5(3) \\ \mathrm{C}(29)-\mathrm{C}(28)-\mathrm{Rh}(1) & 71.18(16) \\ \mathrm{C}(27)-\mathrm{C}(28)-\mathrm{Rh}(1) & 111.22(18) \\ \mathrm{C}(28)-\mathrm{C}(29)-\mathrm{C}(30) & 125.2(3) \\ \mathrm{C}(28)-\mathrm{C}(29)-\mathrm{Rh}(1) & 72.63(15) \\ \mathrm{C}(30)-\mathrm{C}(29)-\mathrm{Rh}(1) & 109.44(19) \\ \mathrm{C}(31)-\mathrm{C}(30)-\mathrm{C}(29) & 114.2(3) \\ \mathrm{C}(30)-\mathrm{C}(31)-\mathrm{C}(32) & 113.7(3) \\ \mathrm{C}(25)-\mathrm{C}(32)-\mathrm{C}(31) & 122.5(3) \\ \mathrm{C}(25)-\mathrm{C}(32)-\mathrm{Rh}(1) & 70.04(16) \\ \mathrm{C}(31)-\mathrm{C}(32)-\mathrm{Rh}(1) & 114.5(2)\end{array}$

Symmetry transformations used to generate equivalent atoms:

Table 4. Anisotropic displacement parameters. $\left(\AA^{2} \times 10^{3}\right)$ for $\mathrm{Rh}(\mathrm{COD})\left(0-\mathrm{C}_{6} \mathrm{H}_{4} P P h_{2} N P h\right)$. The anisotropic displacement factor exponent takes the form: $-2 \pi^{2}\left[h^{2} a^{* 2} U^{11}+\ldots+2 h k a^{*} b^{*} U^{12}\right]$

\begin{tabular}{lllllll}
\hline & $\mathrm{U}^{11}$ & $\mathrm{U}^{22}$ & $\mathrm{U}^{33}$ & $\mathrm{U}^{23}$ & $\mathrm{U}^{13}$ & $\mathrm{U}^{12}$ \\
\hline $\mathrm{Rh}(1)$ & $42(1)$ & $36(1)$ & $30(1)$ & $-5(1)$ & $4(1)$ & $-15(1)$ \\
$\mathrm{N}(1)$ & $53(1)$ & $44(1)$ & $32(1)$ & $-9(1)$ & $5(1)$ & $-24(1)$ \\
$\mathrm{P}(1)$ & $34(1)$ & $37(1)$ & $34(1)$ & $-3(1)$ & $2(1)$ & $-13(1)$ \\
$\mathrm{C}(1)$ & $34(1)$ & $39(1)$ & $-34(1)$ & $-3(1)$ & $-1(1)$ & $-5(1)$ \\
$\mathrm{C}(2)$ & $49(2)$ & $43(2)$ & $37(1)$ & $-5(1)$ & $3(1)$ & $-8(1)$ \\
$\mathrm{C}(3)$ & $54(2)$ & $61(2)$ & $30(1)$ & $-7(1)$ & $4(1)$ & $-1(2)$ \\
$\mathrm{C}(4)$ & $49(2)$ & $65(2)$ & $35(2)$ & $3(1)$ & $10(1)$ & $-9(2)$ \\
$\mathrm{C}(5)$ & $46(2)$ & $50(2)$ & $44(2)$ & $1(1)$ & $9(1)$ & $-18(1)$ \\
$\mathrm{C}(6)$ & $33(1)$ & $44(2)$ & $33(1)$ & $-3(1)$ & $2(1)$ & $-8(1)$ \\
$\mathrm{C}(7)$ & $42(2)$ & $43(2)$ & $35(1)$ & $-5(1)$ & $-1(1)$ & $-6(1)$ \\
$\mathrm{C}(8)$ & $61(2)$ & $43(2)$ & $58(2)$ & $-4(1)$ & $-8(2)$ & $-11(2)$ \\
$\mathrm{C}(9)$ & $107(3)$ & $42(2)$ & $66(2)$ & $-5(2)$ & $-28(2)$ & $-4(2)$
\end{tabular}


(C) 2003 American Chemical Society, Organometallics, Chan om030539g Supporting Info Page 14 Page 14

\begin{tabular}{|c|c|c|c|c|c|c|}
\hline$C(10)$ & $97(3)$ & $66(2)$ & $71(2)$ & $-22(2)$ & $-33(2)$ & $28(2)$ \\
\hline$C(11)$ & $47(2)$ & $113(3)$ & $66(2)$ & $-31(2)$ & $-14(2)$ & $16(2)$ \\
\hline$C(12)$ & $44(2)$ & $73(2)$ & $44(2)$ & $-7(2)$ & $-2(1)$ & $-8(2)$ \\
\hline$C(13)$ & $39(2)$ & $37(1)$ & $43(1)$ & $2(1)$ & $-3(1)$ & $-15(1)$ \\
\hline$C(14)$ & $45(2)$ & $51(2)$ & $53(2)$ & $-6(1)$ & $O(1)$ & $-20(1)$ \\
\hline$C(15)$ & $72(2)$ & $62(2)$ & $61(2)$ & $-9(2)$ & $-8(2)$ & $-36(2)$ \\
\hline$C(16)$ & $55(2)$ & $78(2)$ & $85(2)$ & $0(2)$ & $-21(2)$ & $-36(2)$ \\
\hline$C(17)$ & $37(2)$ & $75(2)$ & $110(3)$ & $-20(2)$ & $-6(2)$ & $-12(2)$ \\
\hline $\mathrm{C}(18)$ & $43(2)$ & $57(2)$ & $78(2)$ & $-15(2)$ & $-3(2)$ & $-13(2)$ \\
\hline$C(19)$ & $56(2)$ & $43(2)$ & $30(1)$ & $-5(1)$ & $4(1)$ & $-28(1)$ \\
\hline$C(20)$ & $60(2)$ & $71(2)$ & $40(2)$ & $-7(1)$ & $3(1)$ & $-26(2)$ \\
\hline$C(21)$ & $78(2)$ & $92(3)$ & $39(2)$ & $-3(2)$ & $-10(2)$ & $-40(2)$ \\
\hline$C(22)$ & $101(3)$ & $77(2)$ & $34(2)$ & $-14(2)$ & $7(2)$ & $-48(2)$ \\
\hline$C(23)$ & $84(2)$ & $53(2)$ & $55(2)$ & $-22(2)$ & $20(2)$ & $-24(2)$ \\
\hline$C(24)$ & $66(2)$ & $50(2)$ & $43(2)$ & $-10(1)$ & $7(1)$ & $-19(2)$ \\
\hline$C(25)$ & $59(2)$ & $53(2)$ & $37(1)$ & $-15(1)$ & $3(1)$ & $-27(2)$ \\
\hline$C(26)$ & $58(2)$ & $85(2)$ & $61(2)$ & $-19(2)$ & $4(2)$ & $-36(2)$ \\
\hline$C(27)$ & $53(2)$ & $63(2)$ & $64(2)$ & $-18(2)$ & $14(2)$ & $-26(2)$ \\
\hline$C(28)$ & $57(2)$ & $50(2)$ & $41(2)$ & $-11(1)$ & $17(1)$ & $-25(2)$ \\
\hline$C(29)$ & $69(2)$ & $57(2)$ & $31(1)$ & $-2(1)$ & $8(1)$ & $-31(2)$ \\
\hline$C(30)$ & $80(2)$ & $55(2)$ & $54(2)$ & $8(2)$ & $5(2)$ & $-20(2)$ \\
\hline$C(31)$ & $108(3)$ & $40(2)$ & $68(2)$ & $-4(2)$ & $14(2)$ & $-21(2)$ \\
\hline$C(32)$ & $72(2)$ & $39(2)$ & $45(2)$ & $-13(1)$ & $13(1)$ & $-20(2)$ \\
\hline
\end{tabular}

Table 5. Hydrogen coordinates $\left(\times 10^{4}\right)$ and isotropic displacement parameters $\left(\AA^{2} \times 10^{3}\right)$ for $\mathrm{Rh}(\mathrm{COD})\left(\mathrm{O}-\mathrm{C}_{6} \mathrm{H}_{4} \mathrm{PPh}_{2} \mathrm{NPh}\right)$

\begin{tabular}{lrrrr}
\hline & $x$ & $y$ & $z$ & $U(e q)$ \\
\hline$H(2 A)$ & 8056 & 5103 & 4988 & 52 \\
$H(3 A)$ & 9082 & 5982 & 3443 & 60 \\
$H(4 A)$ & 9742 & 7835 & 3356 & 62 \\
$H(5 A)$ & 9397 & 8797 & 4865 & 56 \\
$H(8 A)$ & 8309 & 10759 & 6019 & 66 \\
$H(9 A)$ & 6652 & 12493 & 5288 & 89
\end{tabular}


(C) 2003 American Chemical Society, Organometallics, Chan om030539g Supporting Info Page 15

Page 15

\begin{tabular}{|c|c|c|c|c|}
\hline $\mathrm{H}(10 \mathrm{~A})$ & 4319 & 12471 & 5420 & 99 \\
\hline $\mathrm{H}(11 \mathrm{~A})$ & 3626 & 10698 & 6223 & 94 \\
\hline$H(12 A)$ & 5260 & 8936 & 6938 & 66 \\
\hline$H(14 A)$ & 8750 & 9901 & 8348 & 59 \\
\hline $\mathrm{H}(15 \mathrm{~A})$ & 10737 & 10337 & 9023 & 74 \\
\hline$H(16 A)$ & 12911 & 9367 & 8516 & 85 \\
\hline $\mathrm{H}(17 \mathrm{~A})$ & 13125 & 7951 & 7398 & 88 \\
\hline $\mathrm{H}(18 \mathrm{~A})$ & 11171 & 7500 & 6710 & 70 \\
\hline $\mathrm{H}(20 \mathrm{~A})$ & 9421 & 6372 & 9568 & 66 \\
\hline $\mathrm{H}(21 \mathrm{~A})$ & 9269 & 6599 & 11402 & 81 \\
\hline $\mathrm{H}(22 \mathrm{~A})$ & 7327 & 7816 & 12008 & 79 \\
\hline $\mathrm{H}(23 \mathrm{~A})$ & 5543 & 8829 & 10800 & 74 \\
\hline $\mathrm{H}(24 \mathrm{~A})$ & 5664 & 8596 & 8954 & 62 \\
\hline $\mathrm{H}(25 \mathrm{~A})$ & 5923 & 5129 & 5719 & 56 \\
\hline $\mathrm{H}(26 \mathrm{~A})$ & 3828 & 5728 & 6570 & 77 \\
\hline $\mathrm{H}(26 \mathrm{~B})$ & 4073 & 4291 & 6837 & 77 \\
\hline $\mathrm{H}(27 \mathrm{~A})$ & 4354 & 4191 & 8591 & 69 \\
\hline $\mathrm{H}(27 \mathrm{~B})$ & 3420 & 5519 & 8323 & 69 \\
\hline $\mathrm{H}(28 \mathrm{~A})$ & 5112 & 6235 & 8978 & 57 \\
\hline$H(29 A)$ & 7100 & .5097 & 9634 & 61 \\
\hline $\mathrm{H}(30 \mathrm{~A})$ & 8114 & 3163 & 9344 & 78 \\
\hline $\mathrm{H}(30 \mathrm{~B})$ & 6590 & 3020 & 9629 & 78 \\
\hline $\mathrm{H}(31 \mathrm{~A})$ & 6152 & 2772 & 7978 & 86 \\
\hline $\mathrm{H}(31 \mathrm{~B})$ & 7773 & 2327 & 7955 & 86 \\
\hline $\mathrm{H}(32 \mathrm{~A})$ & 7788 & 3789 & 6449 & 60 \\
\hline
\end{tabular}


Table 1. Crystal data and structure refinement for $\mathrm{Rh}(\mathrm{COD})\left(0-\mathrm{C}_{6} \mathrm{H}_{4} \mathrm{PPh}_{2} \mathrm{~N}\left(2,6-\mathrm{Me}_{2} \mathrm{C}_{6} \mathrm{H}_{3}\right)\right)$

Empirical formula

Formula weight

Temperature

Wavelength

Crystal system

Space group

Unit cell dimensions

Volume

Z

Density (calculated)

Absorption coefficient

$\mathrm{F}(000)$

Crystal size

Theta range for data collection

Index ranges

Reflections collected

Independent reflections

Completeness to theta $=23.22^{\circ}$

Refinement method

Data / restraints / parameters

Goodness-of-fit on $\mathrm{F}^{2}$

Final $R$ indices [I $>2$ sigma(I)]

$\mathrm{R}$ indices (all data)

Largest diff. peak and hole
C34 H35 N P Rh

591.51

293(2) K

$0.71073 \AA$

monoclinic

$\mathrm{P} 2(1) / \mathrm{n}$

$\mathrm{a}=10.122(6) \AA$

$\alpha=90^{\circ}$.

$\mathrm{b}=20.056(11) \AA$

$\beta=107.315(11)^{\circ}$.

$\mathrm{c}=14.533(8) \AA$

$\gamma=90^{\circ}$.
4

$1.395 \mathrm{Mg} / \mathrm{m}^{3}$

$0.686 \mathrm{~mm}^{-1}$

1224

$0.29 \times 0.27 \times 0.23 \mathrm{~mm}^{3}$

2.34 to $23.22^{\circ}$.

$-9<=\mathrm{h}<=11,-22<=\mathrm{k}<=20,-16<=\mathrm{l}<=16$

11904

$4018[\mathrm{R}(\mathrm{int})=0.0228]$

$99.6 \%$

Full-matrix least-squares on $\mathrm{F}^{2}$

$4018 / 0 / 334$

0.942

$\mathrm{R} 1=0.0231, \mathrm{wR} 2=0.0611$

$\mathrm{R} 1=0.0331, \mathrm{wR} 2=0.0633$

0.259 and -0.470 e. $\AA^{-3}$ 
(C) 2003 American Chemical Society, Organometallics, Chan om030539g Supporting Info Page 17

Table 2. Atomic coordinates $\left(\times 10^{4}\right)$ and equivalent isotropic displacement parameters $\left(\AA^{2} \times 10^{3}\right)$ for $\mathrm{Rh}(\mathrm{COD})\left(\mathrm{o}-\mathrm{C}_{6} \mathrm{H}_{4} \mathrm{PPh}_{2} \mathrm{~N}\left(2,6-\mathrm{Me}_{2} \mathrm{C}_{6} \mathrm{H}_{3}\right)\right)$. U(eq) is defined as one third of the trace of the orthogonalized Uij tensor.

\begin{tabular}{|c|c|c|c|c|}
\hline & $\mathrm{x}$ & $\mathrm{y}$ & $z$ & $\mathrm{U}(\mathrm{eq})$ \\
\hline $\mathrm{Rh}(1)$ & $1978(1)$ & $1908(1)$ & $-105(1)$ & $37(1)$ \\
\hline$P(1)$ & $426(1)$ & $3048(1)$ & $690(1)$ & $34(1)$ \\
\hline$N(1)$ & $1457(2)$ & $2920(1)$ & $49(2)$ & $36(1)$ \\
\hline$c(1)$ & $649(3)$ & $1696(1)$ & $693(2)$ & $38(1)$ \\
\hline$C(2)$ & $252(3)$ & $1066(1)$ & $967(2)$ & $50(1)$ \\
\hline$C(3)$ & $-665(3)$ & $990(1)$ & $1492(2)$ & $56(1)$ \\
\hline$C(4)$ & $-1232(3)$ & $1534(2)$ & $1823(2)$ & $58(1)$ \\
\hline$C(5)$ & $-867(3)$ & $2161(1)$ & $1599(2)$ & $50(1)$ \\
\hline$C(6)$ & $14(3)$ & $2237(1)$ & $1029(2)$ & $38(1)$ \\
\hline$C(7)^{\circ}$ & $-1126(3)$ & $3494(1)$ & $36(2)$ & $37(1)$ \\
\hline$C(8)$ & $-2411(3)$ & $3180(1)$ & $-242(2)$ & $52(1)$ \\
\hline$C(9)$ & $-3557(3)$ & $3512(2)$ & $-824(2)$ & $65(1)$ \\
\hline$C(10)$ & $-3431(3)$ & $4152(2)$ & $-1128(2)$ & $64(1)$ \\
\hline$C(11)$ & $-2167(3)$ & $4468(1)$ & $-867(2)$ & $52(1)$ \\
\hline$C(12)$ & $-1021(3)$ & $4144(1)$ & $-283(2)$ & $42(1)$ \\
\hline$C(13)$ & $1150(3)$ & $3508(1)$ & $1806(2)$ & $37(1)$ \\
\hline$C(14)$ & $676(3)$ & $4121(1)$ & $2025(2)$ & $47(1)$ \\
\hline$C(15)$ & $1314(4)$ & $4429(1)$ & $2896(2)$ & $61(1)$ \\
\hline$C(16)$ & $2408(4)$ & $4132(2)$ & $3558(2)$ & $60(1)$ \\
\hline$C(17)$ & $2878(4)$ & $3517(2)$ & $3363(2)$ & $63(1)$ \\
\hline$C(18)$ & $2239(3)$ & $3210(1)$ & $2496(2)$ & $57(1)$ \\
\hline$C(19)$ & $1769(3)$ & $3463(1)$ & $-507(2)$ & $36(1)$ \\
\hline$C(20)$ & $933(3)$ & $3557(1)$ & $-146 !(2)$ & $42(1)$ \\
\hline$C(21)$ & $1207(4)$ & $4104(1)$ & $-1976(2)$ & $57(1)$ \\
\hline$C(22)$ & $2277(4)$ & $4531(1)$ & $-1573(3)$ & $68(1)$ \\
\hline$C(23)$ & $3123(4)$ & $4419(1)$ & $-667(3)$ & $62(1)$ \\
\hline$C(24)$ & $2906(3)$ & $3881(1)$ & $-113(2)$ & $44(1)$ \\
\hline$C(25)$ & $-234(3)$ & $3094(1)$ & $-1946(2)$ & $61(1)$ \\
\hline$C(26)$ & $3898(3)$ & $3.773(2)$ & $876(2)$ & $67(1)$ \\
\hline$C(27)$ & $2882(4)$ & $2083(1)$ & $-1313(2)$ & $55(1)$ \\
\hline
\end{tabular}


(C) 2003 American Chemical Society, Organometallics, Chan om030539g Supporting Info Page 18

Page 18

$\begin{array}{rrrrr}C(28) & 3904(3) & 2161(2) & -455(3) & 58(1) \\ \mathrm{C}(29) & 4984(4) & 1650(2) & 29(3) & 82(1) \\ \mathrm{C}(30) & 4423(4) & 961(2) & 59(3) & 77(1) \\ \mathrm{C}(31) & 2946(4) & 964(1) & 125(2) & 57(1) \\ \mathrm{C}(32) & 1772(4) & 946(1) & -682(2) & 54(1) \\ \mathrm{C}(33) & 1783(5) & 945(2) & -1720(2) & 81(1) \\ \mathrm{C}(34) & 2706(4) & 1473(2) & -1955(2) & 74(1)\end{array}$

Table 3. Bond lengths $[\AA]$ and angles $\left[^{\circ}\right]$ for $R h(C O D)\left(o-C_{6} H_{4} P P h_{2} N\left(2,6-M e_{2} C_{6} H_{3}\right)\right)$

\begin{tabular}{ll}
\hline $\mathrm{Rh}(1)-\mathrm{C}(1)$ & $2.066(3)$ \\
$\mathrm{Rh}(1)-\mathrm{C}(32)$ & $2.089(3)$ \\
$\mathrm{Rh}(1)-\mathrm{C}(31)$ & $2.113(3)$ \\
$\mathrm{Rh}(1)-\mathrm{N}(1)$ & $2.125(2)$ \\
$\mathrm{Rh}(1)-\mathrm{C}(28)$ & $2.214(3)$ \\
$\mathrm{Rh}(1)-\mathrm{C}(27)$ & $2.235(3)$ \\
$\mathrm{P}(1)-\mathrm{N}(1)$ & $1.613(2)$ \\
$\mathrm{P}(1)-\mathrm{C}(6)$ & $1.784(2)$ \\
$\mathrm{P}(1)-\mathrm{C}(7)$ & $1.812(3)$ \\
$\mathrm{P}(1)-\mathrm{C}(13)$ & $1.820(3)$ \\
$\mathrm{N}(1)-\mathrm{C}(19)$ & $1.445(3)$ \\
$\mathrm{C}(1)-\mathrm{C}(2)$ & $1.417(3)$ \\
$\mathrm{C}(1)-\mathrm{C}(6)$ & $1.420(4)$ \\
$\mathrm{C}(2)-\mathrm{C}(3)$ & $1.374(4)$ \\
$\mathrm{C}(3)-\mathrm{C}(4)$ & $1.385(4)$ \\
$\mathrm{C}(4)-\mathrm{C}(5)$ & $1.377(4)$ \\
$\mathrm{C}(5)-\mathrm{C}(6)$ & $1.395(4)$ \\
$\mathrm{C}(7)-\mathrm{C}(8)$ & $1.393(4)$ \\
$\mathrm{C}(7)-\mathrm{C}(12)$ & $1.397(3)$ \\
$\mathrm{C}(8)-\mathrm{C}(9)$ & $1.386(4)$ \\
$\mathrm{C}(9)-\mathrm{C}(10)$ & $1.376(4)$ \\
$\mathrm{C}(10)-\mathrm{C}(11)$ & $1.377(4)$ \\
$\mathrm{C}(11)-\mathrm{C}(12)$ & $1.380(4)$ \\
$\mathrm{C}(13)-\mathrm{C}(18)$ & $1.386(4)$ \\
$\mathrm{C}(13)-\mathrm{C}(14)$ & $1.392(3)$ \\
$\mathrm{C}(14)-\mathrm{C}(15)$ & $1.383(4)$ \\
& \\
&
\end{tabular}


(C) 2003 American Chemical Society, Organometallics, Chan om030539g Supporting Info Page 19

\begin{tabular}{|c|c|}
\hline$C(15)-C(16)$ & $1.368(4)$ \\
\hline$C(16)-C(17)$ & $1.382(4)$ \\
\hline$C(17)-C(18)$ & $1.378(4)$ \\
\hline$C(19)-C(24)$ & $1.401(4)$ \\
\hline$C(19)-C(20)$ & $1.406(4)$ \\
\hline$C(20)-C(21)$ & $1.401(4)$ \\
\hline$C(20)-C(25)$ & $1.504(4)$ \\
\hline$C(21)-C(22)$ & $1.369(5)$ \\
\hline$C(22)-C(23)$ & $1.359(5)$ \\
\hline$C(23)-C(24)$ & $1.402(4)$ \\
\hline$C(24)-C(26)$ & $1.504(4)$ \\
\hline$C(27)-C(28)$ & $1.371(4)$ \\
\hline$C(27)-C(34)$ & $1.517(4)$ \\
\hline$C(28)-C(29)$ & $1.511(5)$ \\
\hline$C(29)-C(30)$ & $1.499(5)$ \\
\hline$C(30)-C(31)$ & $1.526(4)$ \\
\hline$C(31)-C(32)$ & $1.400(4)$ \\
\hline$C(32)-C(33)$ & $1.512(4)$ \\
\hline$C(33)-C(34)$ & $1.516(4)$ \\
\hline$C(1)-R h(1)-C(32)$ & $91.79(10)$ \\
\hline$C(1)-R h(1)-C(31)$ & $94.32(11)$ \\
\hline $\mathrm{C}(32)-\mathrm{Rh}(1)-\mathrm{C}(31)$ & $38.92(12)$ \\
\hline$C(1)-\operatorname{Rh}(1)-N(1)$ & $85.08(8)$ \\
\hline$C(32)-\operatorname{Rh}(1)-N(1)$ & $157.76(11)$ \\
\hline$C(31)-\operatorname{Rh}(1)-N(1)$ & $163.21(11)$ \\
\hline$C(1)-\operatorname{Rh}(1)-C(28)$ & $160.22(12)$ \\
\hline$C(32)-\operatorname{Rh}(1)-C(28)$ & $96.23(12)$ \\
\hline$C(31)-R h(1)-C(28)$ & $80.94(12)$ \\
\hline$N(1)-R h(1)-C(28)$ & $93.95(10)$ \\
\hline$C(1)-\operatorname{Rh}(1)-C(27)$ & $163.87(12)$ \\
\hline$C(32)-R h(1)-C(27)$ & $81.08(10)$ \\
\hline$C(31)-R h(1)-C(27)$ & $89.18(11)$ \\
\hline$N(1)-\operatorname{Rh}(1)-C(27)$ & $96.03(9)$ \\
\hline$C(28)-\operatorname{Rh}(1)-C(27)$ & $35.90(11)$ \\
\hline$N(1)-P(1)-C(6)$ & $105.01(11)$ \\
\hline$N(1)-P(1)-C(7)$ & $112.58(12)$ \\
\hline
\end{tabular}


(C) 2003 American Chemical Society, Organometallics, Chan om030539g Supporting Info Page 20

\begin{tabular}{|c|c|}
\hline$C(6)-P(1)-C(7)$ & $110.97(13)$ \\
\hline$N(1)-P(1)-C(13)$ & $115.93(12)$ \\
\hline$C(6)-P(1)-C(13)$ & $106.35(12)$ \\
\hline$C(7)-P(1)-C(13)$ & $105.88(12)$ \\
\hline$C(19)-N(1)-P(1)$ & $119.27(16)$ \\
\hline$C(19)-N(1)-R h(1)$ & $123.97(16)$ \\
\hline$P(1)-N(1)-R h(1)$ & $115.86(10)$ \\
\hline$C(2)-C(1)-C(6)$ & $112.9(2)$ \\
\hline $\mathrm{C}(2)-\mathrm{C}(1)-\mathrm{Rh}(1)$ & $128.9(2)$ \\
\hline$C(6)-C(1)-R h(1)$ & $118.17(17)$ \\
\hline$C(3)-C(2)-C(1)$ & $123.4(3)$ \\
\hline$C(2)-C(3)-C(4)$ & $121.5(2)$ \\
\hline$C(5)-C(4)-C(3)$ & $118.1(3)$ \\
\hline$C(4)-C(5)-C(6)$ & $120.3(3)$ \\
\hline$C(5)-C(6)-C(1)$ & $123.7(2)$ \\
\hline$C(5)-C(6)-P(1)$ & $120.45(19)$ \\
\hline$C(1)-C(6)-P(1)$ & $115.81(19)$ \\
\hline$C(8)-C(7)-C(12)$ & $118.8(3)$ \\
\hline$C(8)-C(7)-P(1)$ & $121.1(2)$ \\
\hline$C(12)-C(7)-P(1)$ & $119.7(2)$ \\
\hline$C(9)-C(8)-C(7)$ & $119.9(3)$ \\
\hline$C(10)-C(9)-C(8)$ & $120.3(3)$ \\
\hline$C(9)-C(10)-C(11)$ & $120.6(3)$ \\
\hline$C(10)-C(11)-C(12)$ & $119.6(3)$ \\
\hline$C(11)-C(12)-C(7)$ & $120.8(3)$ \\
\hline$C(18)-C(13)-C(14)$ & $118.0(3)$ \\
\hline$C(18)-C(13)-P(1)$ & $116.68(19)$ \\
\hline$C(14)-C(13)-P(1)$ & $125.3(2)$ \\
\hline$C(15)-C(14)-C(13)$ & $120.4(3)$ \\
\hline$C(16)-C(15)-C(14)$ & $120.5(3)$ \\
\hline$C(15)-C(16)-C(17)$ & $120.0(3)$ \\
\hline$C(18)-C(17)-C(16)$ & $119.4(3)$ \\
\hline$C(17)-C(18)-C(13)$ & $121.6(3)$ \\
\hline$C(24)-C(19)-C(20)$ & $120.2(2)$ \\
\hline $\mathrm{C}(24)-\mathrm{C}(19)-\mathrm{N}(1)$ & $120.8(2)$ \\
\hline$C(20)-C(19)-N(1)$ & $119.0(2)$ \\
\hline
\end{tabular}


(C) 2003 American Chemical Society, Organometallics, Chan om030539g Supporting Info Page 21

$\begin{array}{lc}\mathrm{C}(21)-\mathrm{C}(20)-\mathrm{C}(19) & 118.4(3) \\ \mathrm{C}(21)-\mathrm{C}(20)-\mathrm{C}(25) & 119.1(3) \\ \mathrm{C}(19)-\mathrm{C}(20)-\mathrm{C}(25) & 122.5(2) \\ \mathrm{C}(22)-\mathrm{C}(21)-\mathrm{C}(20) & 121.2(3) \\ \mathrm{C}(23)-\mathrm{C}(22)-\mathrm{C}(21) & 120.1(3) \\ \mathrm{C}(22)-\mathrm{C}(23)-\mathrm{C}(24) & 121.5(3) \\ \mathrm{C}(19)-\mathrm{C}(24)-\mathrm{C}(23) & 118.4(3) \\ \mathrm{C}(19)-\mathrm{C}(24)-\mathrm{C}(26) & 122.5(2) \\ \mathrm{C}(23)-\mathrm{C}(24)-\mathrm{C}(26) & 119.1(3) \\ \mathrm{C}(28)-\mathrm{C}(27)-\mathrm{C}(34) & 124.7(3) \\ \mathrm{C}(28)-\mathrm{C}(27)-\mathrm{Rh}(1) & 71.23(17) \\ \mathrm{C}(34)-\mathrm{C}(27)-\mathrm{Rh}(1) & 110.85(19) \\ \mathrm{C}(27)-\mathrm{C}(28)-\mathrm{C}(29) & 126.3(3) \\ \mathrm{C}(27)-\mathrm{C}(28)-\mathrm{Rh}(1) & 72.87(17) \\ \mathrm{C}(29)-\mathrm{C}(28)-\mathrm{Rh}(1) & 107.6(2) \\ \mathrm{C}(30)-\mathrm{C}(29)-\mathrm{C}(28) & 114.3(3) \\ \mathrm{C}(29)-\mathrm{C}(30)-\mathrm{C}(31) & 112.6(3) \\ \mathrm{C}(32)-\mathrm{C}(31)-\mathrm{C}(30) & 123.4(3) \\ \mathrm{C}(32)-\mathrm{C}(31)-\mathrm{Rh}(1) & 69.63(16) \\ \mathrm{C}(30)-\mathrm{C}(31)-\mathrm{Rh}(1) & 114.3(2) \\ \mathrm{C}(31)-\mathrm{C}(32)-\mathrm{C}(33) & 125.4(3) \\ \mathrm{C}(31)-\mathrm{C}(32)-\mathrm{Rh}(1) & 71.45(16) \\ \mathrm{C}(33)-\mathrm{C}(32)-\mathrm{Rh}(1) & 111.86(18) \\ \mathrm{C}(32)-\mathrm{C}(33)-\mathrm{C}(34) & 114.3(3) \\ \mathrm{C}(33)-\mathrm{C}(34)-\mathrm{C}(27) & 113.1(2) \\ & \\ & \\ & \end{array}$

Symmetry transformations used to generate equivalent atoms:

Table 4. Anisotropic displacement parameters $\left(\AA^{2} \times 10^{3}\right)$ for $\mathrm{Rh}(\mathrm{COD})\left(0-\mathrm{C}_{6} \mathrm{H}_{4} \mathrm{PPh}_{2} \mathrm{~N}\left(2,6-\mathrm{Me}_{2} \mathrm{C}_{6} \mathrm{H}_{3}\right)\right)$ The anisotropicdisplacement factor exponent takes the form: $-2 \pi^{2}\left[h^{2} a^{* 2} U^{11}+\ldots+2 h k a^{*} b^{*} U^{12}\right]$

\begin{tabular}{lllllll}
\hline & $\mathrm{U}^{11}$ & $\mathrm{U}^{22}$ & $\mathrm{U}^{33}$ & $\mathrm{U}^{23}$ & $\mathrm{U}^{13}$ & $\mathrm{U}^{12}$ \\
\hline $\mathrm{Rh}(1)$ & $49(1)$ & $23(1)$ & $44(1)$ & $0(1)$ & $22(1)$ & $1(1)$ \\
$\mathrm{P}(1)$ & $39(1)$ & $24(1)$ & $41(1)$ & $1(1)$ & $16(1)$ & $0(1)$ \\
$\mathrm{N}(1)$ & $43(1)$ & $24(1)$ & $44(1)$ & $1(1)$ & $19(1)$ & $-1(1)$
\end{tabular}


(C) 2003 American Chemical Society, Organometallics, Chan om030539g Supporting Info Page 22

\begin{tabular}{|c|c|c|c|c|c|c|}
\hline$C(1)$ & $49(2)$ & $29(1)$ & $36(1)$ & $3(1)$ & $14(1)$ & $-3(1)$ \\
\hline$C(2)$ & $75(2)$ & $27(1)$ & $52(2)$ & $4(1)$ & $26(2)$ & $-2(1)$ \\
\hline$C(3)$ & $80(2)$ & $36(2)$ & $57(2)$ & $8(1)$ & $27(2)$ & $-15(2)$ \\
\hline$C(4)$ & $60(2)$ & $61(2)$ & $61(2)$ & $15(2)$ & $31(2)$ & $-11(2)$ \\
\hline$C(5)$ & $60(2)$ & $41(2)$ & $58(2)$ & $7(1)$ & $31(2)$ & $4(1)$ \\
\hline$C(6)$ & $44(2)$ & $30(1)$ & $41(1)$ & $5(1)$ & $15(1)$ & $-2(1)$ \\
\hline$C(7)$ & $39(2)$ & $32(1)$ & $43(2)$ & $-1(1)$ & $15(1)$ & $1(1)$ \\
\hline$C(8)$ & $50(2)$ & $47(2)$ & $59(2)$ & $3(2)$ & $18(2)$ & $-5(2)$ \\
\hline$C(9)$ & $38(2)$ & $80(2)$ & $70(2)$ & $2(2)$ & $6(2)$ & $-7(2)$ \\
\hline$C(10)$ & $50(2)$ & $68(2)$ & $66(2)$ & $11(2)$ & $8(2)$ & $15(2)$ \\
\hline$C(11)$ & $59(2)$ & $42(2)$ & $55(2)$ & $6(1)$ & $16(2)$ & $11(2)$ \\
\hline$C(12)$ & $42(2)$ & $37(2)$ & $46(2)$ & $-1(1)$ & $12(1)$ & $3(1)$ \\
\hline$C(13)$ & $41(2)$ & $31(1)$ & $39(1)$ & $1(1)$ & $14(1)$ & $0(1)$ \\
\hline$C(14)$ & $51(2)$ & $39(2)$ & $48(2)$ & $-3(1)$ & $11(1)$ & $7(1)$ \\
\hline$C(15)$ & $76(2)$ & $44(2)$ & $60(2)$ & $-14(2)$ & $16(2)$ & $6(2)$ \\
\hline$C(16)$ & $74(2)$ & $57(2)$ & $46(2)$ & $-10(2)$ & $11(2)$ & $-6(2)$ \\
\hline$C(17)$ & $66(2)$ & $63(2)$ & $48(2)$ & $2(2)$ & $-I(2)$ & $11(2)$ \\
\hline$C(18)$ & $67(2)$ & $46(2)$ & $55(2)$ & $-1(2)$ & $11(2)$ & $14(2)$ \\
\hline$C(19)$ & $44(2)$ & $23(1)$ & $47(2)$ & $1(1)$ & $24(1)$ & $3(1)$ \\
\hline$C(20)$ & $48(2)$ & $37(2)$ & $50(2)$ & $3(1)$ & $26(2)$ & $5(1)$ \\
\hline $\mathrm{C}(21)$ & $77(2)$ & $47(2)$ & $60(2)$ & $19(2)$ & $39(2)$ & $19(2)$ \\
\hline$C(22)$ & $104(3)$ & $35(2)$ & $94(3)$ & $16(2)$ & $70(3)$ & $5(2)$ \\
\hline$C(23)$ & $73(2)$ & $37(2)$ & $92(3)$ & $-14(2)$ & $51(2)$ & $-18(2)$ \\
\hline$C(24)$ & $48(2)$ & $33(1)$ & $60(2)$ & $-8(1)$ & $29(2)$ & $-7(1)$ \\
\hline$C(25)$ & $63(2)$ & $73(2)$ & $45(2)$ & $-1(2)$ & $15(2)$ & $-3(2)$ \\
\hline$C(26)$ & $50(2)$ & $70(2)$ & $79(2)$ & $-17(2)$ & $17(2)$ & $-17(2)$ \\
\hline$C(27)$ & $80(3)$ & $37(2)$ & $67(2)$ & $4(1)$ & $50(2)$ & $2(2)$ \\
\hline$C(28)$ & $56(2)$ & $42(2)$ & $91(3)$ & $-7(2)$ & $44(2)$ & $-5(2)$ \\
\hline $\mathrm{C}(29)$ & $61(3)$ & $77(2)$ & $113(3)$ & $-10(2)$ & $35(2)$ & $12(2)$ \\
\hline$C(30)$ & $84(3)$ & $69(2)$ & $85(2)$ & $14(2)$ & $37(2)$ & $36(2)$ \\
\hline$C(31)$ & $83(3)$ & $32(2)$ & $69(2)$ & $7(1)$ & $41(2)$ & $15(2)$ \\
\hline$C(32)$ & $90(3)$ & $26(1)$ & $57(2)$ & $-6(1)$ & $39(2)$ & $-6(2)$ \\
\hline C(33) & $135(4)$ & $57(2)$ & $69(2)$ & $-22(2)$ & $57(2)$ & $-25(2)$ \\
\hline$C(34)$ & $111(3)$ & $62(2)$ & $64(2)$ & $-5(2)$ & $52(2)$ & $-5(2)$ \\
\hline
\end{tabular}


(C) 2003 American Chemical Society, Organometallics, Chan om030539g Supporting Info Page 23

Page 23

Table 5. Hydrogen coordinates $\left(\times 10^{4}\right)$ and isotropic displacement parameters $\left(\AA^{2} \times 10^{3}\right)$ for $\mathrm{Rh}(\mathrm{COD})\left(\mathrm{O}-\mathrm{C}_{6} \mathrm{H}_{4} \mathrm{PPh}_{2} \mathrm{~N}\left(2,6-\mathrm{Me}_{2} \mathrm{C}_{6} \mathrm{H}_{3}\right)\right)$.

\begin{tabular}{|c|c|c|c|c|}
\hline & $\mathrm{x}$ & $y$ & $z$ & $\mathrm{U}(\mathrm{eq})$ \\
\hline $\mathrm{H}(2 \mathrm{~A})$ & 631 & 685 & 781 & 60 \\
\hline $\mathrm{H}(3 \mathrm{~A})$ & -910 & 562 & 1628 & 67 \\
\hline $\mathrm{H}(4 \mathrm{~A})$ & -1841 & 1478 & 2186 & 69 \\
\hline $\mathrm{H}(5 \mathrm{~A})$ & -1210 & 2536 & 1829 & 60 \\
\hline $\mathrm{H}(8 \mathrm{~A})$ & -2499 & 2747 & -38 & 62 \\
\hline $\mathrm{H}(9 \mathrm{~A})$ & -4414 & 3301 & -1010 & 77 \\
\hline $\mathrm{H}(10 \mathrm{~A})$ & -4207 & 4373 & -1513 & 76 \\
\hline $\mathrm{H}(11 \mathrm{~A})$ & -2086 & 4899 & -1083 & 63 \\
\hline $\mathrm{H}(12 \mathrm{~A})$ & -170 & 4360 & -99 & 50 \\
\hline $\mathrm{H}(14 \mathrm{~A})$ & -73 & 4325 & 1584 & 57 \\
\hline $\mathrm{H}(15 \mathrm{~A})$ & 996 & 4842 & 3033 & 73 \\
\hline $\mathrm{H}(16 \mathrm{~A})$ & 2836 & 4345 & 4139 & 72 \\
\hline $\mathrm{H}(17 \mathrm{~A})$ & 3618 & 3312 & 3812 & 76 \\
\hline $\mathrm{H}(18 \mathrm{~A})$ & 2547 & 2792 & 2371 & 69 \\
\hline $\mathrm{H}(21 \mathrm{~A})$ & 652 & 4177 & -2604 & 69 \\
\hline $\mathrm{H}(22 \mathrm{~A})$ & 2425 & 4899 & -1920 & 82 \\
\hline $\mathrm{H}(23 \mathrm{~A})$ & 3862 & 4706 & -408 & 74 \\
\hline $\mathrm{H}(25 \mathrm{~A})$ & -295 & 2746 & -1506 & 91 \\
\hline$H(25 B)$ & -66 & 2901 & -2505 & 91. \\
\hline $\mathrm{H}(25 \mathrm{C})$ & -1088 & 3339 & -2137 & 91 \\
\hline $\mathrm{H}(26 \mathrm{~A})$ & 3617 & 3389 & 1164 & 100 \\
\hline $\mathrm{H}(26 \mathrm{~B})$ & 3897 & 4158 & 1268 & 100 \\
\hline $\mathrm{H}(26 \mathrm{C})$ & 4812 & 3703 & 826 & 100 \\
\hline $\mathrm{H}(27 \mathrm{~A})$ & 2584 & 2501 & -1663 & 66 \\
\hline$H(28 A)$ & 4205 & 2623 & -309 & 70 \\
\hline$H(29 A)$ & 5678 & 1633 & -309 & 98 \\
\hline $\mathrm{H}(29 \mathrm{~B})$ & 5440 & 1794 & 683 & 98 \\
\hline $\mathrm{H}(30 \mathrm{~A})$ & 5016 & 725 & 611 & 92 \\
\hline $\mathrm{H}(30 \mathrm{~B})$ & 4442 & 721 & -516 & 92 \\
\hline $\mathrm{H}(31 \mathrm{~A})$ & 2836 & 726 & 686 & 69 \\
\hline
\end{tabular}


(C) 2003 American Chemical Society, Organometallics, Chan om030539g Supporting Info Page 24

Page 24

$\begin{array}{lrrrr}\mathrm{H}(32 \mathrm{~A}) & 999 & 696 & -576 & 65 \\ \mathrm{H}(33 \mathrm{~A}) & 845 & 1012 & -2133 & 98 \\ \mathrm{H}(33 \mathrm{~B}) & 2086 & 511 & -1869 & 98 \\ \mathrm{H}(34 \mathrm{~A}) & 2314 & 1609 & -2621 & 88 \\ \mathrm{H}(34 \mathrm{~B}) & 3609 & 1280 & -1888 & 88\end{array}$

Table 1. Crystal data and structure refinement for Rh(COD) (o$\left.\mathrm{C}_{6} \mathrm{H}_{4} \mathrm{PPh}_{2} \mathrm{~N}\left(3,5-\mathrm{Me}_{2} \mathrm{C}_{6} \mathrm{H}_{3}\right)\right)$. THF.

Empirical formula

Formula weight

Temperature

Wavelength

Crystal system

Space group

Unit cell dimensions

Volume

$\mathrm{Z}$

Density (calculated)

Absorption coefficient

$F(000)$

Crystal size

Theta range for data collection

Index ranges

Reflections collected

Independent reflections

Completeness to theta $=23.20^{\circ}$

Refinement method

Data / restraints / parameters

Goodness-of-fit on $\mathrm{F}^{2}$

Final $\mathrm{R}$ indices [ $\mathrm{I}>2 \operatorname{sigma}(\mathrm{I})]$

$\mathrm{R}$ indices (all data)

Largest diff. peak and hole
C38 H43 N O P Rh

663.61

293(2) K

$0.71073 \AA$

monoclinic

$\mathrm{P} 2(1) / \mathrm{c}$

$\mathrm{a}=21.924(12) \AA$

$\alpha=90^{\circ}$.

$\mathrm{b}=15.092(8) \AA$

$\beta=95.361(10)^{\circ}$.

$c=10.022(5) \AA$

$\gamma=90^{\circ}$.

$3301(3) \AA^{3}$

4.

$1.335 \mathrm{Mg} / \mathrm{m}^{3}$

$0.596 \mathrm{~mm}^{-1}$

1384

$0.27 \times 0.22 \times 0.17 \mathrm{~mm}^{3}$

2.30 to $23.20^{\circ}$.

$-24<=\mathrm{h}<=22,-14<=\mathrm{k}<=16,-1 \mid<=\mathrm{l}<=11$

13754

$4690[\mathrm{R}($ int $)=0.0184]$

$99.5 \%$

Full-matrix least-squares on $\mathrm{F}^{2}$

$4690 / 0 / 379$

0.830

$\mathrm{R} 1=0.0269, \mathrm{wR} 2=0.0915$

$\mathrm{R} 1=0.0339, \mathrm{wR} 2=0.0982$

0.367 and -0.460 e. $\AA^{-3}$ 
(C) 2003 American Chemical Society, Organometallics, Chan om030539g Supporting Info Page 25

Table 2. Atomic coordinates $\left(\times 10^{4}\right)$ and equivalent isotropic displacement parameters $\left(\AA^{2} \times 10^{3}\right)$ for $\mathrm{Rh}(\mathrm{COD})\left(\mathrm{O}-\mathrm{C}_{6} \mathrm{H}_{4} \mathrm{PPh}_{2} \mathrm{~N}\left(3,5-\mathrm{Me}_{2} \mathrm{C}_{6} \mathrm{H}_{3}\right)\right)$. THF. $\mathrm{U}(\mathrm{eq})$ is defined as one third of the trace of the orthogonalized $U^{i j}$ tensor.

\begin{tabular}{|c|c|c|c|c|}
\hline & $x$ & $\mathrm{y}$ & $z$ & $\mathrm{U}(\mathrm{eq})$ \\
\hline $\mathrm{Rh}(1)$ & $7073(1)$ & $5226(1)$ & $3450(1)$ & $33(1)$ \\
\hline$P(1)$ & $7733(1)$ & $5050(1)$ & $787(1)$ & $34(1)$ \\
\hline$N(1)$ & $7071(1)$ & $5179(2)$ & $1350(2)$ & $37(1)$ \\
\hline$C(1)$ & $8013(1)$ & $5110(2)$ & $3467(3)$ & $37(1)$ \\
\hline$C(2)$ & $8459(2)$ & $5086(2)$ & $4590(3)$ & $51(1)$ \\
\hline$C(3)$ & $9076(2)$ & $5065(3)$ & $4475(4)$ & $68(1)$ \\
\hline$C(4)$ & $9310(2)$ & $5072(3)$ & $3246(4)$ & $72(1)$ \\
\hline$C(5)$ & $8899(2)$ & $5083(3)$ & $2103(4)$ & $59(1)$ \\
\hline$C(6)$ & $8274(1)$ & $5095(2)$ & $2237(3)$ & $37(1)$ \\
\hline$C(7)$ & $7933(1)$ & $5897(2)$ & $-387(3)$ & $38(1)$ \\
\hline$C(8)$ & $8359(1)$ & $5734(2)$ & $-1307(3)$ & $50(1)$ \\
\hline$C(9)$ & $8539(2)$ & $6422(2)$ & $-2105(3)$ & $59(1)$ \\
\hline$C(10)$ & $8305(2)$ & $7261(2)$ & $-1994(3)$ & $64(1)$ \\
\hline$C(11)$ & $7878(2)$ & $7425(2)$ & $-1092(4)$ & $66(1)$ \\
\hline$C(12)$ & $7702(1)$ & $6748(2)$ & $-276(3)$ & $50(1)$ \\
\hline$C(13)$ & $7809(1)$ & $3999(2)$ & $-54(3)$ & $38(1)$ \\
\hline$C(14)$ & $7492(1)$ & $3857(2)$ & $-1312(3)$ & $46(1)$ \\
\hline$C(15)$ & $7454(2)$ & $3013(2)$ & $-1843(3)$ & $56(1)$ \\
\hline$C(16)$ & $7717(2)$ & $2306(2)$ & $-1164(3)$ & $59(1)$ \\
\hline$C(17)$ & $8045(2)$ & $2440(2)$ & $68(3)$ & $59(1)$ \\
\hline$C(18)$ & $8091(1)$ & $3279(2)$ & $629(3)$ & $47(1)$ \\
\hline$C(19)$ & $6530(1)$ & $5012(2)$ & $425(3)$ & $36(1)$ \\
\hline$C(20)$ & $6319(1)$ & $4153(2)$ & $195(3)$ & $45(1)$ \\
\hline$C(21)$ & $5813(1)$ & $3980(2)$ & $-711(3)$ & $50(1)$ \\
\hline$C(22)$ & $5506(2)$ & $4690(2)$ & $-1360(3)$ & $5 J(1)$ \\
\hline$C(23)$ & $5696(1)$ & $5556(2)$ & $-1122(3)$ & $45(1)$ \\
\hline$C(24)$ & $6216(1)$ & $5709(2)$ & $-230(3)$ & $39(1)$ \\
\hline$C(25)$ & $5611(2)$ & $3031(3)$ & $-1015(5)$ & $80(1)$ \\
\hline$C(26)$ & $5347(2)$ & $6316(3)$ & $-1819(3)$ & $61(1)$ \\
\hline$C(27)$ & $6184(1)$ & $5905(2)$ & $3374(3)$ & $49(1)$ \\
\hline
\end{tabular}


(C) 2003 American Chemical Society, Organometallics, Chan om030539g Supporting Info Page 26

Page 26

$\begin{array}{lrrrr}C(28) & 6202(2) & 6558(3) & 4517(4) & 66(1) \\ C(29) & 6584(2) & 6244(2) & 5784(3) & 61(1) \\ C(30) & 7108(1) & 5629(2) & 5465(3) & 46(1) \\ C(31) & 7063(2) & 4708(2) & 5380(3) & 44(1) \\ C(32) & 6485(1) & 4175(2) & 5525(3) & 54(1) \\ C(33) & 5913(2) & 4553(2) & 4734(3) & 54(1) \\ C(34) & 6065(1) & 5011(2) & 3463(3) & 47(1) \\ C(35) & 10293(6) & 7446(7) & 1769(10) & 238(5) \\ C(36) & 10654(3) & 6624(7) & 2030(7) & 149(3) \\ C(37) & 10459(2) & 5975(4) & 1173(6) & 96(1) \\ C(38) & 10120(4) & 6355(5) & 195(7) & 146(2) \\ O(1) & 9840(4) & 7200(7) & 667(10) & 275(4)\end{array}$

Table

3. Bond lengths $[\AA]$ and angles $\left[{ }^{\circ}\right]$ for $\mathrm{Rh}(\mathrm{COD})\left(\mathrm{O}-\mathrm{C}_{6} \mathrm{H}_{4} \mathrm{PPh}_{2} \mathrm{~N}\left(3,5-\mathrm{Me}_{2} \mathrm{C}_{6} \mathrm{H}_{3}\right)\right)$. THF

\begin{tabular}{ll}
\hline $\mathrm{Rh}(1)-\mathrm{C}(1)$ & $2.068(3)$ \\
$\mathrm{Rh}(1)-\mathrm{C}(31)$ & $2.089(3)$ \\
$\mathrm{Rh}(1)-\mathrm{C}(30)$ & $2.104(3)$ \\
$\mathrm{Rh}(1)-\mathrm{N}(1)$ & $2.105(3)$ \\
$\mathrm{Rh}(1)-\mathrm{C}(27)$ & $2.196(3)$ \\
$\mathrm{Rh}(1)-\mathrm{C}(34)$ & $2.235(3)$ \\
$\mathrm{P}(1)-\mathrm{N}(1)$ & $1.616(3)$ \\
$\mathrm{P}(1)-\mathrm{C}(6)$ & $1.790(3)$ \\
$\mathrm{P}(1)-\mathrm{C}(13)$ & $1.811(3)$ \\
$\mathrm{P}(1)-\mathrm{C}(7)$ & $1.819(3)$ \\
$\mathrm{N}(1)-\mathrm{C}(19)$ & $1.459(4)$ \\
$\mathrm{C}(1)-\mathrm{C}(6)$ & $1.407(4)$ \\
$\mathrm{C}(1)-\mathrm{C}(2)$ & $1.420(4)$ \\
$\mathrm{C}(2)-\mathrm{C}(3)$ & $1.367(5)$ \\
$\mathrm{C}(3)-\mathrm{C}(4)$ & $1.378(5)$ \\
$\mathrm{C}(4)-\mathrm{C}(5)$ & $1.389(5)$ \\
$\mathrm{C}(5)-\mathrm{C}(6)$ & $1.389(5)$ \\
$\mathrm{C}(7)-\mathrm{C}(12)$ & $1.389(4)$ \\
$\mathrm{C}(7)-\mathrm{C}(8)$ & $1.395(4)$ \\
$\mathrm{C}(8)-\mathrm{C}(9)$ & $1.390(4)$ \\
$\mathrm{C}(9)-\mathrm{C}(10)$ & $1.374(5)$ \\
&
\end{tabular}


(C) 2003 American Chemical Society, Organometallics, Chan om030539g Supporting Info Page 27

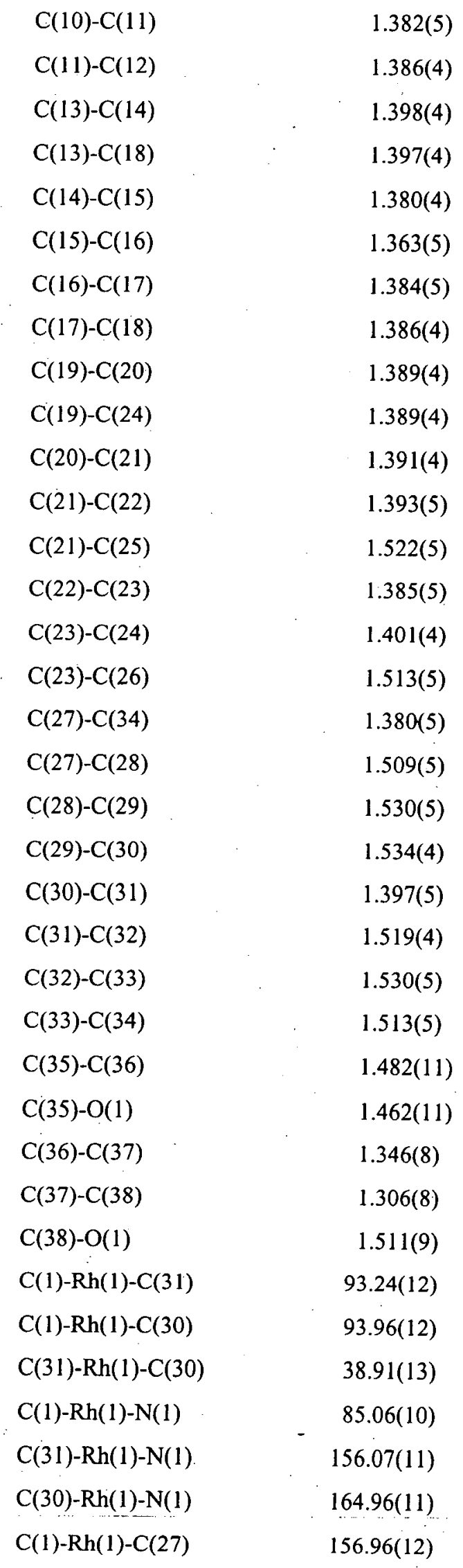


(C) 2003 American Chemical Society, Organometallics, Chan om030539g Supporting Info Page 28

\begin{tabular}{|c|c|}
\hline$C(31)-R h(1)-C(27)$ & $96.98(12)$ \\
\hline$C(30)-\operatorname{Rh}(1)-C(27)$ & $81.44(12)$ \\
\hline$N(1)-R h(1)-C(27)$ & $93.60(10)$ \\
\hline$C(1)-\operatorname{Rh}(1)-C(34)$ & $166.75(14)$ \\
\hline$C(31)-R h(1)-C(34)$ & $81.08(12)$ \\
\hline$C(30)-R h(1)-C(34)$ & $89.10(12)$ \\
\hline $\mathrm{N}(1)-\mathrm{Rh}(1)-\mathrm{C}(34)$ & $95.28(10)$ \\
\hline$C(27)-R h(1)-C(34)$ & $36.29(12)$ \\
\hline$N(1)-P(1)-C(6)$ & $105.06(14)$ \\
\hline$N(1)-P(1)-C(13)$ & $113.20(13)$ \\
\hline$C(6)-P(1)-C(13)$ & $109.16(13)$ \\
\hline$N(1)-P(1)-C(7)$ & $114.80(13)$ \\
\hline$C(6)-P(1)-C(7)$ & $108.42(13)$ \\
\hline$C(13)-P(1)-C(7)$ & $106.06(13)$ \\
\hline$C(19)-N(1)-P(1)$ & $117.4(2)$ \\
\hline$C(19)-\mathrm{N}(1)-\mathrm{Rh}(1)$ & $124.47(19)$ \\
\hline$P(1)-N(1)-R h(1)$ & $115.85(13)$ \\
\hline$C(6)-C(1)-C(2)$ & $112.8(3)$ \\
\hline$C(6)-C(1)-\operatorname{Rh}(1)$ & $118.7(2)$ \\
\hline$C(2)-C(1)-R h(1)$ & $128.4(2)$ \\
\hline$C(3)-C(2)-C(1)$ & $123.1(3)$ \\
\hline$C(2)-C(3)-C(4)$ & $121.9(3)$ \\
\hline$C(3)-C(4)-C(5)$ & $118.0(3)$ \\
\hline$C(4)-C(5)-C(6)$ & $119.4(4)$ \\
\hline$C(5)-C(6)-C(1)$ & $124.7(3)$ \\
\hline$C(5)-C(6)-P(1)$ & $120.4(3)$ \\
\hline$C(1)-C(6)-P(1)$ & $114.8(2)$ \\
\hline$C(12)-C(7)-C(8)$ & $1 \cdot 19.2(3)$ \\
\hline$C(12)-C(7)-P(1)$ & $119.1(2)$ \\
\hline$C(8)-C(7)-P(1)$ & $121.4(2)$ \\
\hline$C(9)-C(8)-C(7)$ & $119.5(3)$ \\
\hline$C(10)-C(9)-C(8)$ & $120.8(3)$ \\
\hline$C(9)-C(10)-C(11)$ & $119.9(3)$ \\
\hline$C(10)-C(11)-C(12)$ & $119.8(3)$ \\
\hline$C(11)-C(12)-C(7)$ & $120.6(3)$ \\
\hline$C(14)-C(13)-C(18)$ & $1] 8.9(3)$ \\
\hline
\end{tabular}


(C) 2003 American Chemical Society, Organometallics, Chan om030539g Supporting Info Page 29

\begin{tabular}{|c|c|}
\hline$C(14)-C(13)-P(1)$ & $119.6(2)$ \\
\hline$C(18)-C(13)-P(1)$ & $120.6(2)$ \\
\hline$C(15)-C(14)-C(13)$ & $119.7(3)$ \\
\hline$C(16)-C(15)-C(14)$ & $121.5(3)$ \\
\hline$C(15)-C(16)-C(17)$ & $119.4(3)$ \\
\hline$C(16)-C(17)-C(18)$ & $120.5(3)$ \\
\hline$C(17)-C(18)-C(13)$ & $120.0(3)$ \\
\hline$C(20)-C(19)-C(24)$ & $119.1(3)$ \\
\hline$C(20)-C(19)-N(1)$ & $120.4(3)$ \\
\hline$C(24)-C(19)-N(1)$ & $120.5(3)$ \\
\hline$C(19)-C(20)-C(21)$ & $121.1(3)$ \\
\hline$C(22)-C(21)-C(20)$ & $118.8(3)$ \\
\hline$C(22)-C(21)-C(25)$ & $120.8(3)$ \\
\hline$C(20)-C(21)-C(25)$ & $120.4(3)$ \\
\hline $\mathrm{C}(23)-\mathrm{C}(22)-\mathrm{C}(21)$ & $121.4(3)$ \\
\hline$C(22)-C(23)-C(24)$ & $118.6(3)$ \\
\hline$C(22)-C(23)-C(26)$ & $120.4(3)$ \\
\hline$C(24)-C(23)-C(26)$ & $121.0(3)$ \\
\hline$C(19)-C(24)-C(23)$ & $121.0(3)$ \\
\hline$C(34)-C(27)-C(28)$ & $125.6(3)$ \\
\hline $\mathrm{C}(34)-\mathrm{C}(27)-\mathrm{Rh}(1)$ & $73.39(18)$ \\
\hline$C(28)-C(27)-R h(1)$ & $108.6(2)$ \\
\hline$C(27)-C(28)-C(29)$ & $113.6(3)$ \\
\hline$C(30)-C(29)-C(28)$ & $112.2(3)$ \\
\hline$C(31)-C(30)-C(29)$ & $124.4(3)$ \\
\hline$C(31)-C(30)-R h(1)$ & $69.96(16)$ \\
\hline$C(29)-C(30)-R h(1)$ & $114.4(2)$ \\
\hline$C(30)-C(31)-C(32)$ & $125.2(3)$ \\
\hline$C(30)-C(31)-R h(1)$ & $71.12(16)$ \\
\hline$C(32)-C(31)-R h(1)$ & $111.5(2)$ \\
\hline$C(31)-C(32)-C(33)$ & $113.8(3)$ \\
\hline$C(34)-C(33)-C(32)$ & $111.9(3)$ \\
\hline$C(27)-C(34)-C(33)$ & $124.0(3)$ \\
\hline$C(27)-C(34)-R h(1)$ & $70.33(17)$ \\
\hline$C(33)-C(34)-R h(1)$ & $111.5(2)$ \\
\hline $\mathrm{C}(36)-\mathrm{C}(35)-\mathrm{O}(1)$ & $103.6(7)$ \\
\hline
\end{tabular}


(C) 2003 American Chemical Society, Organometallics, Chan om030539g Supporting Info Page 30

Page 30

$\begin{array}{ll}C(37)-C(36)-C(35) & 111.4(6) \\ C(38)-C(37)-C(36) & 106.5(6) \\ C(37)-C(38)-O(1) & 110.8(7) \\ C(35)-O(1)-C(38) & 100.5(7)\end{array}$

Symmetry transformations used to generate equivalent atoms:

Table 4. Anisotropic displacement parameters $\left(\AA^{2} \times 10^{3}\right)$ for $\mathrm{Rh}(\mathrm{COD})\left(\mathrm{O}-\mathrm{C}_{6} \mathrm{H}_{4} \mathrm{PPh}_{2} \mathrm{~N}(3,5-\right.$ $\left.\left.\mathrm{Me}_{2} \mathrm{C}_{6} \mathrm{H}_{3}\right)\right)$.THF.. The anisotropic displacement factor exponent takes the form: $-2 \pi^{2}\left[h^{2} \mathrm{a}^{* 2} \mathrm{U}^{11}+\ldots+2\right.$ $h \mathrm{ka}^{*} \mathrm{~b}^{*} \mathrm{U}^{12}$ ]

\begin{tabular}{|c|c|c|c|c|c|c|}
\hline & $\mathrm{U}^{11}$ & $\mathrm{U}^{22}$ & $U^{33}$ & $\mathrm{U}^{23}$ & $U^{13}$ & $\mathrm{U}^{12}$ \\
\hline $\operatorname{Rh}(1)$ & $32(1)$ & $38(1)$ & $28(1)$ & $-1(1)$ & $-2(1)$ & I(1) \\
\hline$P(1)$ & $33(1)$ & $38(1)$ & $29(1)$ & $0(1)$ & $-1(1)$ & $O(1)$ \\
\hline$N(1)$ & $32(1)$ & $47(2)$ & $32(1)$ & $-1(1)$ & $-4(1)$ & $O(1)$ \\
\hline$C(1)$ & $37(2)$ & $36(2)$ & $35(2)$ & $1(1)$ & $-6(1)$ & $-3(1)$ \\
\hline$C(2)$ & $43(2)$ & $71(2)$ & $35(2)$ & $-2(2)$ & $-6(1)$ & $-2(2)$ \\
\hline$C(3)$ & $45(2)$ & $104(3)$ & $50(2)$ & $3(2)$ & $-21(2)$ & $-5(2)$ \\
\hline$C(4)$ & $29(2)$ & $125(4)$ & $59(2)$ & $6(2)$ & $-7(2)$ & $-1(2)$ \\
\hline$C(5)$ & $38(2)$ & $88(3)$ & $48(2)$ & $5(2)$ & $-3(2)$ & $-4(2)$ \\
\hline$C(6)$ & $33(2)$ & $4 I(2)$ & $36(2)$ & $1(1)$ & $-3(1)$ & $-1(1)$ \\
\hline$C(7)$ & $39(2)$ & $40(2)$ & $32(1)$ & $0(1)$ & $-3(1)$ & $-5(1)$ \\
\hline $\mathrm{C}(8)$ & $58(2)$ & $47(2)$ & $45(2)$ & $-3(2)$ & $11(1)$ & $-5(2)$ \\
\hline$C(9)$ & $68(2)$ & $65(2)$ & $47(2)$ & $4(2)$ & $17(2)$ & $-14(2)$ \\
\hline$C(10)$ & $75(2)$ & $59(2)$ & $59(2)$ & $17(2)$ & $5(2)$ & $-20(2)$ \\
\hline$C(11)$ & $77(3)$ & $39(2)$ & $81(3)$ & $12(2)$ & $4(2)$ & $-2(2)$ \\
\hline$C(12)$ & $51(2)$ & $44(2)$ & $55(2)$ & $2(2)$ & $8(2)$ & $-2(2)$ \\
\hline$C(13)$ & $39(2)$ & $40(2)$ & $35(2)$ & (1) & $7(1)$ & $1(1)$ \\
\hline$C(14)$ & $58(2)$ & $43(2)$ & $36(2)$ & $1(1)$ & $3(1)$ & $3(1)$ \\
\hline$C(15)$ & $76(2)$ & $53(2)$ & $40(2)$ & $-10(2)$ & $5(2)$ & $-2(2)$ \\
\hline$C(16)$ & $90(3)$ & $39(2)$ & $53(2)$ & $-6(2)$ & $22(2)$ & $-1(2)$ \\
\hline$C(17)$ & $83(3)$ & $41(2)$ & $55(2)$ & $10(2)$ & $16(2)$ & $12(2)$ \\
\hline$C(18)$ & $.56(2)$ & $46(2)$ & $39(2)$ & $6(1)$ & $6(1)$ & $5(2)$ \\
\hline$C(19)$ & $31(2)$ & $46(2)$ & $30(2)$ & $-3(1)$ & $0(1)$ & $0(1)$ \\
\hline$C(20)$ & $41(2)$ & $46(2)$ & $45(2)$ & $1(1)$ & $-1(1)$ & $-3(1)$ \\
\hline$C(21)$ & $42(2)$ & $57(2)$ & $51(2)$ & $-12(2)$ & $0(1)$ & $-10(2)$ \\
\hline
\end{tabular}


(C) 2003 American Chemical Society, Organometallics, Chan om030539g Supporting Info Page 31 rage 31

$\begin{array}{lcccccc}\mathrm{C}(22) & 33(2) & 69(2) & 49(2) & -10(2) & -7(1) & -6(2) \\ \mathrm{C}(23) & 34(2) & 61(2) & 39(2) & 0(2) & -1(1) & 0(2) \\ \mathrm{C}(24) & 35(2) & 45(2) & 38(2) & 0(1) & -1(1) & -4(1) \\ \mathrm{C}(25) & 69(3) & 66(3) & 101(3) & -26(2) & -11(2) & -18(2) \\ \mathrm{C}(26) & 47(2) & 74(3) & 59(2) & 11(2) & -13(2) & 8(2) \\ \mathrm{C}(27) & 38(2) & 59(2) & 48(2) & 3(2) & 1(1) & 14(2) \\ \mathrm{C}(28) & 69(2) & 59(2) & 74(2) & -4(2) & 16(2) & 19(2) \\ \mathrm{C}(29) & 67(2) & 65(2) & 53(2) & -19(2) & 13(2) & 2(2) \\ \mathrm{C}(30) & 44(2) & 61(2) & 33(2) & -7(2) & 1(1) & -4(2) \\ \mathrm{C}(31) & 44(2) & 59(2) & 29(2) & 3(1) & -1(1) & 2(1) \\ \mathrm{C}(32) & 52(2) & 62(2) & 48(2) & 10(2) & 6(2) & -6(2) \\ \mathrm{C}(33) & 43(2) & 66(2) & 53(2) & 0(2) & 6(2) & -7(2) \\ \mathrm{C}(34) & 30(2) & 69(2) & 40(2) & -1(2) & -2(1) & 2(2) \\ \mathrm{C}(35) & 362(16) & 175(9) & 166(8) & -88(7) & -27(9) & 41(10) \\ \mathrm{C}(36) & 122(5) & 192(8) & 131(6) & 12(6) & -7(4) & -27(6) \\ \mathrm{C}(37) & 77(3) & 99(4) & 112(4) & 1(4) & 13(3) & 12(3) \\ \mathrm{C}(38) & 172(7) & 123(6) & 149(7) & -19(5) & 43(6) & 6(5) \\ \mathrm{O}(1) & 301(9) & 282(10) & 236(7) & -11(8) & -16(7) & 114(8) \\ & & & & & & \end{array}$

Table 5.

Hydrogen coordinates $\left(\mathrm{x} 10^{4}\right)$ and isotropic displacement parameters $\left(\AA^{2} \times 10^{3}\right)$

for $\mathrm{Rh}(\mathrm{COD})\left(\mathrm{O}-\mathrm{C}_{6} \mathrm{H}_{4} \mathrm{PPh}_{2} \mathrm{~N}\left(3,5-\mathrm{Me}_{2} \mathrm{C}_{6} \mathrm{H}_{3}\right)\right)$. THF..

$\begin{array}{llll}\text { x } & \text { y } & \text { z } & \text { U(eq) }\end{array}$

$\begin{array}{lrrrr}\mathrm{H}(2 \mathrm{~A}) & 8326 & 5084 & 5444 & 61 \\ \mathrm{H}(3 \mathrm{~A}) & 9345 & 5046 & 5248 & 82 \\ \mathrm{H}(4 \mathrm{~A}) & 9730 & 5070 & 3183 & 86 \\ \mathrm{H}(5 \mathrm{~A}) & 9041 & 5081 & 1257 & 70 \\ \mathrm{H}(8 \mathrm{~A}) & 8522 & 5169 & -1386 & 60 \\ \mathrm{H}(9 \mathrm{~A}) & 8822 & 6313 & -2722 & 71 \\ \mathrm{H}(10 \mathrm{~A}) & 8433 & 7717 & -2524 & 77 \\ \mathrm{H}(11 \mathrm{~A}) & 7710 & 7988 & -1034 & 79 \\ \mathrm{H}(12 \mathrm{~A}) & 7426 & 6866 & 352 & 60 \\ \mathrm{H}(14 \mathrm{~A}) & 7308 & 4330 & -1789 & 55 \\ \mathrm{H}(15 \mathrm{~A}) & 7245 & 2924 & -2683 & 67\end{array}$


(C) 2003 American Chemical Society, Organometallics, Chan om030539g Supporting Info Page 32 Page 32

\begin{tabular}{|c|c|c|c|c|}
\hline$H(16 A)$ & 7676 & 1739 & -1525 & 71 \\
\hline$H(17 A)$ & 8236 & 1963 & 522. & 71 \\
\hline $\mathrm{H}(18 \mathrm{~A})$ & 8309 & 3363 & 1461 & 56 \\
\hline $\mathrm{H}(20 \mathrm{~A})$ & 6519 & 3685 & 655 & 54 \\
\hline $\mathrm{H}(22 \mathrm{~A})$ & 5167 & 4580 & -1965 & 61 \\
\hline$H(24 A)$ & 6353 & 6286 & -75 & 47 \\
\hline $\mathrm{H}(25 \mathrm{~A})$ & 5260 & .3032 & -1665 & 120 \\
\hline$H(25 B)$ & 5505 & 2751 & -207 & 120 \\
\hline$H(25 C)$ & 5939 & 2710 & -1364 & 120 \\
\hline $\mathrm{H}(26 \mathrm{~A})$ & 5538 & 6866 & -1540 & 92 \\
\hline$H(26 B)$ & 4932 & 6312 & -1587 & 92 \\
\hline$H(26 C)$ & 5349 & 6252 & -2771 & 92 \\
\hline $\mathrm{H}(27 \mathrm{~A})$ & 6052 & 6164 & 2498 & 58 \\
\hline $\mathrm{H}(28 \mathrm{~A})$ & 5786 & 6670 & 4731 & 80 \\
\hline $\mathrm{H}(28 \mathrm{~B})$ & 6369 & 7115 & 4229 & 80 \\
\hline $\mathrm{H}(29 \mathrm{~A})$ & 6753 & 6755 & 6276 & 73 \\
\hline $\mathrm{H}(29 \mathrm{~B})$ & 6320 & 5932 & 6353 & 73 \\
\hline$H(30 A)$ & 7513 & 5839 & 5827 & 56 \\
\hline $\mathrm{H}(31 \mathrm{~A})$ & 7441 & 4397 & 5703 & 53 \\
\hline $\mathrm{H}(32 \mathrm{~A})$ & 6416 & 4150 & 6466 & 65 \\
\hline $\mathrm{H}(32 \mathrm{~B})$ & 6548 & 3573 & 5226 & 65 \\
\hline $\mathrm{H}(33 \mathrm{~A})$ & 5625 & 4076 & 4506 & 65 \\
\hline $\mathrm{H}(33 \mathrm{~B})$ & 5718 & 4973 & 5291 & 65 \\
\hline$H(34 A)$ & 5868 & 4748 & 2637 & 56 \\
\hline $\mathrm{H}(35 \mathrm{~A})$ & 10551 & 7927 & 1509 & 285 \\
\hline$H(35 B)$ & 10096 & 7623 & 2554 & 285 \\
\hline$H(36 A)$ & 11083 & 6746 & 1940 & 179 \\
\hline $\mathrm{H}(36 \mathrm{~B})$ & 10618 & 6429 & 2941 & 179 \\
\hline $\mathrm{H}(37 \mathrm{~A})$ & 10805 & 5678 & 833 & 115 \\
\hline $\mathrm{H}(37 \mathrm{~B})$ & 10222 & 5541 & 1618 & 115 \\
\hline $\mathrm{H}(38 \mathrm{~A})$ & 9797 & 5952 & -142 & 176 \\
\hline $\mathrm{H}(38 \mathrm{~B})$ & 10368 & 6488 & -531 & 176 \\
\hline
\end{tabular}


Table 1. Crystal data and structure refinement for (COD) $\left(0-\mathrm{C}_{6} \mathrm{H}_{4} \mathrm{PPh}_{2} \mathrm{~N}\left(2,6-i-\mathrm{Pr}_{2} \mathrm{C}_{6} \mathrm{H}_{3}\right)\right.$ )

Empirical formula

Formula weight

Temperature

Wavelength

Crystal system

Space group

Unit cell dimensions

Volume

Z

Density (calculated)

Absorption coefficient

$\mathrm{F}(000)$

Crystal size

Theta range for data collection

Index ranges

Reflections collected

Independent reflections

Completeness to theta $=23.32^{\circ}$

Refinement method

Data / restraints / parameters

Goodness-of-fit on $\mathrm{F}^{2}$

Final $\mathrm{R}$ indices [ $\mathrm{I}>2 \operatorname{sigma}(\mathrm{I})]$

$\mathrm{R}$ indices (all data)

Largest diff. peak and hole

\section{C38 H43 N P Rh}

647.61

293(2) K

$0.71073 \AA$

triclinic

P-1

$$
\begin{array}{ll}
\mathrm{a}=9.927(5) \AA & \alpha=89.621(10)^{\circ} . \\
\mathrm{b}=11.193(6) \AA & \beta=85.158(9)^{\circ} . \\
\mathrm{c}=15.544(8) \AA & \gamma=72.044(10)^{\circ} .
\end{array}
$$

1636.7(15) $\AA^{3}$

2

$1.314 \mathrm{Mg} / \mathrm{m}^{3}$

$0.597 \mathrm{~mm}^{-1}$

676

$0.28 \times 0.23 \times 0.18 \mathrm{~mm}^{3}$

2.30 to $23.32^{\circ}$.

$-8<=\mathrm{h}<=11,-12<=\mathrm{k}<=11,-17<=\mathrm{k}<=16$

7043

$4655[\mathrm{R}(\mathrm{int})=0.0134]$

$98.2 \%$

Full-matrix least-squares on $\mathrm{F}^{2}$

$4655 / 0 / 370$

0.929

$\mathrm{R} 1=0.0244, \mathrm{wR} 2=0.0630$

$\mathrm{R} 1=0.0303, \mathrm{wR} 2=0.0641$

0.459 and -0.381 e. $\AA^{-3}$ 
(C) 2003 American Chemical Society, Organometallics, Chan om030539g Supporting Info Page 34

Page 34

Table 2. Atomic coordinates $\left(\times 10^{4}\right)$ and equivalent isotropic displacement parameters $\left(\AA^{2} \times 10^{3}\right)$ for $(C O D)\left(o-C_{6} H_{4} P P h_{2} N\left(2,6-i-P_{2} C_{6} H_{3}\right)\right)$. U(eq) is defined as one third of the trace of the orthogonalized $U^{\mathrm{ij}}$ tensor.

\begin{tabular}{|c|c|c|c|c|}
\hline & $\mathrm{x}$ & $y$ & $z$ & $\mathrm{U}(\mathrm{eq})$ \\
\hline $\operatorname{Rh}(1)$ & $6973(1)$ & $9160(1)$ & $8232(1)$ & $32(1)$ \\
\hline$P(1)$ & $5278(1)$ & $7941(1)$ & $7092(1)$ & $29(1)$ \\
\hline $\mathrm{N}(1)$ & $6851(2)$ & $7706(2)$ & $7383(1)$ & $30(1)$ \\
\hline$C(1)$ & $5095(3)$ & $10216(2)$ & $7775(1)$ & $33(1)$ \\
\hline$C(2)$ & $4321(3)$ & $11510(2)$ & $7937(2)$ & $43(1)$ \\
\hline$C(3)$ & $2963(3)$ & $12043(2)$ & $7708(2)$ & $49(1)$ \\
\hline$C(4)$ & $2251(3)$ & $11339(2)$ & $7316(2)$ & $49(1)$ \\
\hline$C(5)$ & $2943(3)$ & $10086(2)$ & $7136(2)$ & $44(1)$ \\
\hline$C(6)$ & $4339(3)$ & $9562(2)$ & $7337(1)$ & $33(1)$ \\
\hline$C(7)$ & $4221(3)$ & $7088(2)$ & $7699(2)$ & $35(1)$ \\
\hline$C(8)$ & $.3368(3)$ & $6489(2)$ & $7329(2)$ & $45(1)$ \\
\hline$C(9)$ & $2561(3)$ & 5893(3) & $7840(2)$ & $61(1)$ \\
\hline$C(10)$ & $2598(4)$ & $5906(3)$ & $8723(2)$ & $73(1)$ \\
\hline$C(11)$ & $3409(4)$ & $6516(3)$ & $9097(2)$ & $69(1)$ \\
\hline$C(12)$ & $4225(3)$ & $7116(3)$ & $8594(2)$ & $49(1)$ \\
\hline$C(13)$ & $5234(3)$ & $7569(2)$ & $5957(1)$ & $34(1)$ \\
\hline$C(14)$ & $4663(3)$ & $8495(3)$ & $5376(2)$ & $50(1)$ \\
\hline$C(15)$ & $4694(4)$ & $8190(3)$ & $4509(2)$ & $65(1)$ \\
\hline$C(16)$ & $5305(4)$ & $6977(3)$ & $4214(2)$ & $65(1)$ \\
\hline$C(17)$ & $5901(3)$ & $6048(3)$ & $4775(2)$ & $56(1)$ \\
\hline$C(18)$ & $5873(3)$ & $6342(3)$ & $5647(2)$ & $45(1)$ \\
\hline$C(19)$ & $8071(2)$ & $6747(2)$ & $6991(1)$ & $31(1)$ \\
\hline$C(20)$ & $8452(3)$ & $5496(2)$ & $7281(2)$ & $38(1)$ \\
\hline$C(21)$ & $9615(3)$ & $4612(2)$ & $6845(2)$ & $51(1)$ \\
\hline$C(22)$ & $10419(3)$ & $4919(3)$ & $6173(2)$ & $56(1)$ \\
\hline$C(23)$ & $10080(3)$ & $6155(3)$ & $5919(2)$ & $49(1)$ \\
\hline$C(24)$ & $8918(3)$ & $7077(2)$ & $6307(1)$ & $36(1)$ \\
\hline$C(25)$ & $7667(3)$ & $5057(2)$ & $8039(2)$ & $50(1)$ \\
\hline$C(26)$ & $8688(4)$ & $4404(3)$ & $8717(2)$ & $74(1)$ \\
\hline$C(27)$ & $6868(4)$ & $4165(3)$ & $7754(2)$ & $73(1)$ \\
\hline
\end{tabular}


(C) 2003 American Chemical Society, Organometallics, Chan om030539g Supporting Info Page 35

Page 35

$\begin{array}{lrrrr}C(28) & 8608(3) & 8419(3) & 5985(2) & 49(1) \\ C(29) & 8440(4) & 8495(3) & 5017(2) & 67(1) \\ C(30) & 9778(5) & 8983(3) & 6188(2) & 86(1) \\ C(31) & 8300(3) & 7804(2) & 9071(2) & 44(1) \\ C(32) & 9204(3) & 8218(3) & 8515(2) & 45(1) \\ C(33) & 9934(3) & 9167(3) & 8759(2) & 59(1) \\ C(34) & 9072(3) & 10515(3) & 8577(2) & 61(1) \\ C(35) & 7487(3) & 10736(2) & 8649(2) & 47(1) \\ C(36) & 6708(3) & 10337(2) & 9335(2) & 45(1) \\ C(37) & 7375(3) & 9664(3) & 10119(2) & 55(1) \\ C(38) & 7844(3) & 8241(3) & 9998(2) & 57(1)\end{array}$

3. Bond lengths $[\AA]$ and angles $\left[^{\circ}\right]$ for $(\mathrm{COD})\left(0-\mathrm{C}_{6} \mathrm{H}_{4} \mathrm{PPh}_{2} \mathrm{~N}\left(2,6-i-\mathrm{Pr}_{2} \mathrm{C}_{6} \mathrm{H}_{3}\right)\right)$

\begin{tabular}{ll}
\hline $\mathrm{Rh}(1)-\mathrm{C}(1)$ & $2.057(3)$ \\
$\mathrm{Rh}(1)-\mathrm{C}(35)$ & $2.103(3)$ \\
$\mathrm{Rh}(1)-\mathrm{C}(36)$ & $2.117(3)$ \\
$\mathrm{Rh}(1)-\mathrm{N}(1)$ & $2.140(2)$ \\
$\mathrm{Rh}(1)-\mathrm{C}(31)$ & $2.184(3)$ \\
$\mathrm{Rh}(1)-\mathrm{C}(32)$ & $2.220(3)$ \\
$\mathrm{P}(1)-\mathrm{N}(1)$ & $1.605(2)$ \\
$\mathrm{P}(1)-\mathrm{C}(6)$ & $1.792(2)$ \\
$\mathrm{P}(1)-\mathrm{C}(13)$ & $1.822(2)$ \\
$\mathrm{P}(1)-\mathrm{C}(7)$ & $1.829(2)$ \\
$\mathrm{N}(1)-\mathrm{C}(19)$ & $1.440(3)$ \\
$\mathrm{C}(1)-\mathrm{C}(6)$ & $1.411(3)$ \\
$\mathrm{C}(1)-\mathrm{C}(2)$ & $1.427(3)$ \\
$\mathrm{C}(2)-\mathrm{C}(3)$ & $1.372(4)$ \\
$\mathrm{C}(3)-\mathrm{C}(4)$ & $1.384(4)$ \\
$\mathrm{C}(4)-\mathrm{C}(5)$ & $1.377(4)$ \\
$\mathrm{C}(5)-\mathrm{C}(6)$ & $1.390(4)$ \\
$\mathrm{C}(7)-\mathrm{C}(8)$ & $1.391(4)$ \\
$\mathrm{C}(7)-\mathrm{C}(12)$ & $1.392(4)$ \\
$\mathrm{C}(8)-\mathrm{C}(9)$ & $1.390(4)$ \\
$\mathrm{C}(9)-\mathrm{C}(10)$ & $1.376(5)$ \\
$\mathrm{C}(10)-\mathrm{C}(11)$ & $1.370(5)$ \\
& \\
\hline &
\end{tabular}


(C) 2003 American Chemical Society, Organometallics, Chan om030539g Supporting Info Page 36

\begin{tabular}{|c|c|}
\hline$C(11)-C(12)$ & $1.393(4)$ \\
\hline$C(13)-C(14)$ & $1.387(3)$ \\
\hline$C(13)-C(18)$ & $1.393(4)$ \\
\hline$C(14)-C(15)$ & $1.389(4)$ \\
\hline$C(15)-C(16)$ & $1.370(4)$ \\
\hline$C(16)-C(17)$ & $1.374(4)$ \\
\hline$C(17)-C(18)$ & $1.394(4)$ \\
\hline$C(19)-C(20)$ & $1.414(3)$ \\
\hline$C(19)-C(24)$ & $1.418(3)$ \\
\hline$C(20)-C(21)$ & $1.394(4)$ \\
\hline$C(20)-C(25)$ & $1.520(4)$ \\
\hline$C(21)-C(22)$ & $1.367(4)$ \\
\hline$C(22)-C(23)$ & $1.382(4)$ \\
\hline$C(23)-C(24)$ & $1.384(4)$ \\
\hline$C(24)-C(28)$ & $1.528(4)$ \\
\hline$C(25)-C(26)$ & $1.544(4)$ \\
\hline$C(25)-C(27)$ & $1.541(4)$ \\
\hline$C(28)-C(29)$ & $1.528(4)$ \\
\hline$C(28)-C(30)$ & $1.537(5)$ \\
\hline$C(31)-C(32)$ & $1.374(4)$ \\
\hline$C(31)-C(38)$ & $1.511(4)$ \\
\hline$C(32)-C(33)$ & $1.524(4)$ \\
\hline$C(33)-C(34)$ & $1.525(4)$ \\
\hline$C(34)-C(35)$ & $1.510(4)$ \\
\hline$C(35)-C(36)$ & $1.415(4)$ \\
\hline$C(36)-C(37)$ & $1.519(4)$ \\
\hline$C(37)-C(38)$ & $1.523(4)$ \\
\hline$C(1)-R h(1)-C(35)$ & $93.83(11)$ \\
\hline$C(1)-\operatorname{Rh}(1)-C(36)$ & $93.55(10)$ \\
\hline$C(35)-\operatorname{Rh}(1)-C(36)$ & $39.17(10)$ \\
\hline$C(1)-R h(1)-N(1)$ & $85.54(9)$ \\
\hline$C(35)-R h(1)-N(1)$ & $158.83(9)$ \\
\hline $\mathrm{C}(36)-\mathrm{Rh}(1)-\mathrm{N}(1)$ & $161.98(9)$ \\
\hline$C(1)-R h(1)-C(31)$ & $155.60(10)$ \\
\hline $\mathrm{C}(35)-\mathrm{Rh}(1)-\mathrm{C}(31)$ & $96.81(11)$ \\
\hline$C(36)-R h(1)-C(31)$ & $81.24(11)$ \\
\hline
\end{tabular}


(C) 2003 American Chemical Society, Organometallics, Chan om030539g Supporting Info Page 37

\begin{tabular}{|c|c|}
\hline$N(1)-R h(1)-C(31)$ & $92.17(9)$ \\
\hline$C(1)-R h(1)-C(32)$ & $168.07(10)$ \\
\hline$C(35)-R h(1)-C(32)$ & $80.93(11)$ \\
\hline $\mathrm{C}(36)-\mathrm{Rh}(1)-\mathrm{C}(32)$ & $89.12(11)$ \\
\hline$N(1)-R h(1)-C(32)$ & $95.47(9)$ \\
\hline$C(31)-R h(1)-C(32)$ & $36.33(10)$ \\
\hline$N(1)-P(1)-C(6)$ & $105.98(11)$ \\
\hline$N(1)-P(1)-C(13)$ & $113.91(11)$ \\
\hline$C(6)-P(1)-C(13)$ & $111.15(11)$ \\
\hline$N(1)-P(1)-C(7)$ & $115.27(11)$ \\
\hline$C(6)-P(1)-C(7)$ & $104.27(11)$ \\
\hline$C(13)-P(1)-C(7)$ & $105.90(11)$ \\
\hline$C(19)-N(1)-P(1)$ & $122.67(16)$ \\
\hline$C(19)-N(1)-R h(1)$ & $124.00(15)$ \\
\hline$P(1)-N(1)-R h(1)$ & $112.45(10)$ \\
\hline$C(6)-C(1)-C(2)$ & $113.3(2)$ \\
\hline$C(6)-C(1)-R h(1)$ & $116.88(17)$ \\
\hline$C(2)-C(1)-R h(1)$ & $129.17(18)$ \\
\hline$C(3)-C(2)-C(1)$ & $122.8(2)$ \\
\hline$C(2)-C(3)-C(4)$ & $121.3(2)$ \\
\hline$C(5)-C(4)-C(3)$ & $118.8(3)$ \\
\hline$C(4)-C(5)-C(6)$ & $119.6(3)$ \\
\hline$C(5)-C(6)-C(1)$ & $124.1(2)$ \\
\hline$C(5)-C(6)-P(1)$ & $120.62(19)$ \\
\hline$C(1)-C(6)-P(1)$ & $115.26(18)$ \\
\hline$C(8)-C(7)-C(12)$ & $119.1(2)$ \\
\hline$C(8)-C(7)-P(1)$ & $124.31(19)$ \\
\hline$C(12)-C(7)-P(1)$ & $116.48(19)$ \\
\hline$C(9)-C(8)-C(7)$ & $120.7(3)$ \\
\hline$C(10)-C(9)-C(8)$ & $119.6(3)$ \\
\hline$C(11)-C(10)-C(9)$ & $120.2(3)$ \\
\hline$C(10)-C(11)-C(12)$ & $120.9(3)$ \\
\hline$C(7)-C(12)-C(11)$ & $119.4(3)$ \\
\hline$C(14)-C(13)-C(18)$ & $118.7(2)$ \\
\hline$C(14)-C(13)-P(1)$ & $121.5(2)$ \\
\hline$C(18)-C(13)-P(1)$ & $119.65(18)$ \\
\hline
\end{tabular}


(C) 2003 American Chemical Society, Organometallics, Chan om030539g Supporting Info Page 38

\begin{tabular}{|c|c|}
\hline$C(15)-C(14)-C(13)$ & $120.2(3)$ \\
\hline$C(16)-C(15)-C(14)$ & $120.6(3)$ \\
\hline$C(15)-C(16)-C(17)$ & $120.1(3)$ \\
\hline$C(16)-C(17)-C(18)$ & $119.8(3)$ \\
\hline$C(17)-C(18)-C(13)$ & $120.5(3)$ \\
\hline$C(20)-C(19)-C(24)$ & $119.6(2)$ \\
\hline $\mathrm{C}(20)-\mathrm{C}(19)-\mathrm{N}(1)$ & $121.6(2)$ \\
\hline$C(24)-C(19)-N(1)$ & $118.8(2)$ \\
\hline$C(21)-C(20)-C(19)$ & $118.1(2)$ \\
\hline$C(21)-C(20)-C(25)$ & $118.0(2)$ \\
\hline$C(19)-C(20)-C(25)$ & $123.9(2)$ \\
\hline$C(22)-C(21)-C(20)$ & $122.6(2)$ \\
\hline$C(21)-C(22)-C(23)$ & $119.0(3)$ \\
\hline$C(22)-C(23)-C(24)$ & $121.6(3)$ \\
\hline$C(23)-C(24)-C(19)$ & $119.1(2)$ \\
\hline$C(23)-C(24)-C(28)$ & $118.4(2)$ \\
\hline$C(19)-C(24)-C(28)$ & $122.5(2)$ \\
\hline$C(20)-C(25)-C(26)$ & $111.4(3)$ \\
\hline$C(20)-C(25)-C(27)$ & $112.3(3)$ \\
\hline$C(26)-C(25)-C(27)$ & $109.3(2)$ \\
\hline$C(29)-C(28)-C(24)$ & $112.1(2)$ \\
\hline$C(29)-C(28)-C(30)$ & $109.3(2)$ \\
\hline$C(24)-C(28)-C(30)$ & $111.4(2)$ \\
\hline$C(32)-C(31)-C(38)$ & $125.7(3)$ \\
\hline$C(32)-C(31)-\operatorname{Rh}(1)$ & $73.25(15)$ \\
\hline$C(38)-C(31)-R h(1)$ & $108.58(18)$ \\
\hline$C(31)-C(32)-C(33)$ & $124.4(3)$ \\
\hline$C(31)-C(32)-R h(1)$ & $70.42(15)$ \\
\hline $\mathrm{C}(33)-\mathrm{C}(32)-\mathrm{Rh}(1)$ & $111.51(19)$ \\
\hline$C(32)-C(33)-C(34)$ & $112.5(2)$ \\
\hline$C(35)-C(34)-C(33)$ & $113.6(2)$ \\
\hline$C(36)-C(35)-C(34)$ & $125.3(3)$ \\
\hline$C(36)-C(35)-R h(1)$ & $70.96(15)$ \\
\hline$C(34)-C(35)-R h(1)$ & $112.00(19)$ \\
\hline$C(35)-C(36)-C(37)$ & $123.4(3)$ \\
\hline$C(35)-C(36)-R h(1)$ & $69.87(15)$ \\
\hline
\end{tabular}


(C) 2003 American Chemical Society, Organometallics, Chan om030539g Supporting Info Page 39

Page 39

$\begin{array}{ll}\mathrm{C}(37)-\mathrm{C}(36)-\mathrm{Rh}(1) & 114.31(18) \\ \mathrm{C}(36)-\mathrm{C}(37)-\mathrm{C}(38) & 112.1(2) \\ \mathrm{C}(31)-\mathrm{C}(38)-\mathrm{C}(37) & 113.5(2)\end{array}$

Symmetry transformations used to generate equivalent atoms:

Table 4. Anisotropic displacement parameters $\left(\AA^{2} \times 10^{3}\right)$ for $(\mathrm{COD})\left(0-\mathrm{C}_{6} \mathrm{H}_{4} \mathrm{PPh}_{2} \mathrm{~N}\left(2,6-i-\mathrm{Pr}_{2} \mathrm{C}_{6} \mathrm{H}_{3}\right)\right)$

. The anisotropic displacement factor exponent takes the form: $-2 \pi^{2}\left[h^{2} a^{* 2} U^{11}+\ldots+2 h k a^{*} b^{*} U^{12}\right]$

\begin{tabular}{lcccccc}
\hline & $\mathrm{U}^{11}$ & $\mathrm{U}^{22}$ & $\mathrm{U}^{33}$ & $\mathrm{U}^{23}$ & $\mathrm{U}^{13}$ & $\mathrm{U}^{12}$ \\
\hline $\mathrm{Rh}(1)$ & $34(1)$ & $33(1)$ & $31(1)$ & $-1(1)$ & $-6(1)$ & $-11(1)$ \\
$\mathrm{P}(1)$ & $31(1)$ & $29(1)$ & $28(1)$ & $0(1)$ & $-4(1)$ & $-9(1)$ \\
$\mathrm{N}(1)$ & $31(1)$ & $29(1)$ & $29(1)$ & $-1(1)$ & $-4(1)$ & $-8(1)$ \\
$\mathrm{C}(1)$ & $39(1)$ & $32(1)$ & $27(1)$ & $2(1)$ & $-1(1)$ & $-8(1)$ \\
$\mathrm{C}(2)$ & $53(2)$ & $35(1)$ & $39(1)$ & $-1(1)$ & $-5(1)$ & $-11(1)$ \\
$\mathrm{C}(3)$ & $54(2)$ & $31(1)$ & $48(2)$ & $4(1)$ & $0(1)$ & $5(1)$ \\
$\mathrm{C}(4)$ & $40(2)$ & $43(2)$ & $53(2)$ & $5(1)$ & $-7(1)$ & $4(1)$ \\
$\mathrm{C}(5)$ & $40(2)$ & $43(2)$ & $47(2)$ & $2(1)$ & $-9(1)$ & $-8(1)$ \\
$\mathrm{C}(6)$ & $34(1)$ & $30(1)$ & $30(1)$ & $3(1)$ & $-3(1)$ & $-5(1)$ \\
$\mathrm{C}(7)$ & $33(1)$ & $35(1)$ & $36(1)$ & $3(1)$ & $-1(1)$ & $-9(1)$ \\
$\mathrm{C}(8)$ & $45(2)$ & $48(2)$ & $46(2)$ & $1(1)$ & $-4(1)$ & $-18(1)$ \\
$\mathrm{C}(9)$ & $54(2)$ & $67(2)$ & $75(2)$ & $5(2)$ & $-5(2)$ & $-36(2)$ \\
$\mathrm{C}(10)$ & $69(2)$ & $90(3)$ & $74(2)$ & $22(2)$ & $5(2)$ & $-46(2)$ \\
$\mathrm{C}(11)$ & $71(2)$ & $99(3)$ & $44(2)$ & $15(2)$ & $3(2)$ & $-41(2)$ \\
$\mathrm{C}(12)$ & $47(2)$ & $64(2)$ & $40(2)$ & $1(1)$ & $-2(1)$ & $-24(1)$ \\
$\mathrm{C}(13)$ & $35(1)$ & $42(1)$ & $29(1)$ & $0(1)$ & $-5(1)$ & $-17(1)$ \\
$\mathrm{C}(14)$ & $61(2)$ & $51(2)$ & $39(2)$ & $4(1)$ & $-12(1)$ & $-15(1)$ \\
$\mathrm{C}(15)$ & $85(2)$ & $79(2)$ & $38(2)$ & $11(2)$ & $-20(2)$ & $-31(2)$ \\
$\mathrm{C}(16)$ & $85(2)$ & $94(3)$ & $31(2)$ & $-7(2)$ & $-3(2)$ & $-52(2)$ \\
$\mathrm{C}(17)$ & $65(2)$ & $61(2)$ & $46(2)$ & $-20(2)$ & $12(2)$ & $-31(2)$ \\
$\mathrm{C}(18)$ & $53(2)$ & $47(2)$ & $39(1)$ & $-3(1)$ & $0(1)$ & $-20(1)$ \\
$\mathrm{C}(19)$ & $29(1)$ & $33(1)$ & $29(1)$ & $-3(1)$ & $-7(1)$ & $-7(1)$ \\
$\mathrm{C}(20)$ & $40(2)$ & $32(1)$ & $41(1)$ & $-1(1)$ & $-8(1)$ & $-7(1)$ \\
$\mathrm{C}(21)$ & $49(2)$ & $34(2)$ & $62(2)$ & $1(1)$ & $-11(2)$ & $2(1)$ \\
$\mathrm{C}(22)$ & $43(2)$ & $53(2)$ & $58(2)$ & $-17(2)$ & $3(1)$ & $5(1)$ \\
$\mathrm{C}(23)$ & $44(2)$ & $62(2)$ & $38(1)$ & $-4(1)$ & $3(1)$ & $-12(2)$ \\
& & & & & & \\
\hline
\end{tabular}


(C) 2003 American Chemical Society, Organometallics, Chan om030539g Supporting Info Page 40 Page 40

$\begin{array}{lllllll}\mathrm{C}(24) & 37(1) & 42(1) & 29(1) & -2(1) & -4(1) & -11(1) \\ \mathrm{C}(25) & 54(2) & 37(2) & 56(2) & 12(1) & -3(1) & -10(1) \\ \mathrm{C}(26) & 86(3) & 60(2) & 66(2) & 24(2) & -12(2) & -10(2) \\ \mathrm{C}(27) & 78(2) & 48(2) & 98(3) & 14(2) & -5(2) & -27(2) \\ \mathrm{C}(28) & 54(2) & 47(2) & 43(2) & 6(1) & 10(1) & -16(1) \\ \mathrm{C}(29) & 66(2) & 79(2) & 60(2) & 27(2) & -17(2) & -26(2) \\ \mathrm{C}(30) & 140(4) & 86(3) & 62(2) & 20(2) & -22(2) & -73(3) \\ \mathrm{C}(31) & 46(2) & 39(2) & 45(2) & 2(1) & -18(1) & -6(1) \\ \mathrm{C}(32) & 37(2) & 49(2) & 46(2) & -7(1) & -12(1) & -5(1) \\ \mathrm{C}(33) & 43(2) & 85(2) & 57(2) & -7(2) & -9(1) & -28(2) \\ \mathrm{C}(34) & 65(2) & 68(2) & 66(2) & -2(2) & -13(2) & -41(2) \\ \mathrm{C}(35) & 55(2) & 38(2) & 54(2) & -7(1) & -11(1) & -22(1) \\ \mathrm{C}(36) & 50(2) & 42(2) & 43(2) & -13(1) & -6(1) & -15(1) \\ \mathrm{C}(37) & 61(2) & 72(2) & 38(2) & -9(1) & -6(1) & -26(2) \\ \mathrm{C}(38) & 61(2) & 69(2) & 42(2) & 8(1) & -16(1) & -17(2)\end{array}$

Table 5 .

Hydrogen coordinates $\left(\times 10^{4}\right)$ and isotropic displacement parameters $\left(\AA^{2} \times 10^{3}\right)$

for $(\mathrm{COD})\left(\mathrm{O}-\mathrm{C}_{6} \mathrm{H}_{4} \mathrm{PPh}_{2} \mathrm{~N}\left(2,6-i-\mathrm{Pr}_{2} \mathrm{C}_{6} \mathrm{H}_{3}\right)\right) \mathrm{j}$.

\begin{tabular}{lcccc}
\hline & $x$ & $y$ & $z$ & $U(e q)$ \\
\hline$H(2 A)$ & & & & \\
$H(3 A)$ & 4755 & 12012 & 8210 & 51 \\
$H(4 A)$ & 2511 & 12895 & 7819 & 58 \\
$H(5 A)$ & 1323 & 11706 & 7176 & 59 \\
$H(8 A)$ & 2479 & 9592 & 6881 & 53 \\
$H(9 A)$ & 3337 & 6486 & 6732 & 54 \\
$H(10 A)$ & 2000 & 5488 & 7588 & 73 \\
$H(11 A)$ & 2069 & 5499 & 9067 & 88 \\
$H(12 A)$ & 3416 & 6529 & 9695 & 82 \\
$H(14 A)$ & 4768 & 7533 & 8854 & 58 \\
$H(15 A)$ & 4257 & 9324 & 5568 & 60 \\
$H(16 A)$ & 4298 & 8815 & 4125 & 78 \\
$H(17 A)$ & 5315 & 6781 & 3632 & 78 \\
$H(18 A)$ & 6322 & 5225 & 4573 & 67 \\
& 6284 & 5714 & 6026 & 54
\end{tabular}

